\title{
From Confining Fields on the Lattice to Higher Dimensions in the Continuum
}

\author{
V. I. Zakharov \\ Istituto Nazionale di Fisica Nucleare - Sezione di Pisa, Largo Pontecorvo, 3, 56127 Pisa, Italy; and \\ Max-Planck Institut für Physik, Föhringer Ring 6, 80805, München, Germany
}

(Received on 13 October, 2006)

\begin{abstract}
We discuss the relation between lattice phenomenology of confining fields in the vacuum state of Yang-Mills theories (mostly SU(2) case) and continuum theories. In the continuum, understanding of the confinement is most straightforward in the dual formulation which involves higher dimensions. We try to bridge these two approaches to the confinement, let it be on a rudimentary level. We review lattice data on low-dimensional vacuum defects, like monopoles, center vortices. There is certain resemblance to dual strings, domain walls considered in the continuum version of Yang-Mills theories.
\end{abstract}

Keywords: Confinement; Extra dimensions

\section{OUTLINE OF THE REVIEW}

\section{A. Strings takeover}

This review is based on three lectures given at the conference "Infrared QCD in Rio" in June of the 2006. My primary aim was to review the lattice data on confining fields in lattice Yang-Mills theories. There is accumulating data on the lattice supporting the idea that it is lattice strings that are responsible for the confinement.

On the continuum side, strings are becoming more and more popular as well. It seems even fair to label this year as time of strings takeover. I do feel that QCD issues are more discussed now in terms of strings than in terms of quarks and gluons.

For example, the last Conference in the Minneapolis series is full of discussion of strings from various perspectives:

I. Klebanov on QCD and strings [1],

J. Erdmenger and J. Sonnenschein on applications of AdS/QCD, see [2] and [3], respectively,

D. Tong on string-like solutions in field theory [4],

among others ${ }^{1}$

First, I had an idea to confront all the continuum-theory approaches with the lattice data. But it turns too much an ambitious program. Nevertheless, I hope that the reader will develop certain feelings. For example, that the lattice is producing evidence in favor of AdS/QCD correspondence which is, (unlike the AdS/CFT correspondence) appears a pure speculation theoretically. As for the classical solutions, which always delegate a crucial role to scalar fields, they seem not to apply directly to lattice data since there is no Higgs field in pure gauge theories which we will discuss. Nevertheless, the resulting picture for the 'solitons' bears a striking resemblance to the lattice data on the 'vacuum defects'.

Coming back to our blitz-overview of the literature, I found that the most popular, 'hot' problem recently was calculation - with a look at the RHIC data- of the drag force acting on a

${ }^{1}$ Transparencies of the talks can be found on the cite of the conference CAQCD06. We give references to similar papers in the archive. quark moving through quark-gluon plasma, see in particular [5].

Very schematically, the quark moves in our space with velocity $v, x=v \cdot t$, and there is a string attached to it which extends from into an extra dimension $z$ so that coordinate of the string is

$$
x(t, z)=v t+\xi(z) .
$$

The drag force is due to resistance of the medium to the string. The action of the string is the Nambu-Goto action:

$$
S=\frac{1}{2 \pi \alpha^{\prime}} \int d^{2} \sigma \sqrt{\operatorname{det} g_{\alpha \beta}}
$$

where

$g_{\alpha \beta} \equiv G_{\mu \nu} \partial_{\alpha} X^{\mu} \partial_{\beta} X^{\nu}, \alpha=1,2(o r, x, t) ; \mu, v=1, \ldots, 10$.

Finally, the metrics $G_{\mu \nu}$ of the $10 \mathrm{~d}$ space can be read from:

$$
d s_{10}^{2}=H^{-1 / 2}\left(-h d t^{2}+d \mathbf{x}^{2}\right)+H^{1 / 2}\left(\frac{d z^{2}}{h}+d \Omega_{5}^{2}\right),
$$

where

$$
H=1+\frac{L^{4}}{z^{4}}, h=1-\frac{z_{H}^{4}}{z^{4}}
$$

The drag force is calculable by minimizing the classical string action and in this sense the scheme is very simple conceptually. The price is introduction of quite a few new notions ( $z$ as the fifth coordinate, $d \Omega_{5}^{2}$ as $5 \mathrm{~d}$ sphere living in extra coordinates as well, $z_{H}$ as a horizon, so that $z \leq z_{H}, L$ as a new dimensional constant). In this particular problem the only relevant new parameter is the horizon which, in fact, is reducible to the temperature.

\section{B. Stakes are high}

I would like to emphasize one point which is not so commonly mentioned. Namely, strings mean now fundamental, or infinitely thin strings. In this respect, discovering strings now is like discovering quarks about 30 years ago. Strings can be 
thought of as quarks of non-perturbative QCD. It is an absolutely new turn: it is the physics of infrared which is being discussed but the strings are rather fundamental than 'effective'.

This point is somewhat obscured by the assumption that strings live in extra dimensions. Thus, strings are fundamental, but fundamental in extra dimensions. To decide whether they remain fundamental in $4 \mathrm{~d}$ one should develop a picture for extra dimensions. Without going into any detail now, let us mention that we consider extra dimensions as means to describe physics in the same $4 \mathrm{~d}^{2}$.

Like quarks, strings can be used also for constructing models which are not sensitive to the UV scale. Checking these models we do not check the fundamental nature of the strings. There is an analogy to constituent quarks. For example, calculation of the drag force mentioned above deals mostly with 'effective' strings since there is no direct probe of the thickness of the string ${ }^{3}$. Applications of models exclusively in the IR (infrared) do not allow to distinguish between fundamental and effective strings.

Thus, there is no much (if at all) discussion of the question, what is an analog to deep-inelastic scattering in case of strings. We will argue that in fact - at least as a matter of principlelattices do allow to probe the point-like (better to say, linelike) nature of strings. Indeed, lattice measurements allow to probe distances down to lattice spacing $a$. We will discuss the issue in much more detail later.

Thus, the stakes are high: lattice can probe consistency of strings on the quantum level while continuum-theory applications of strings rest on a mere assumption that classical approximation is valid.

\section{Matching strings and lattices}

In principle, one can learn everything about nonperturbative QCD through lattice measurements. In particular, the string picture can be tested.

The main, and great at that, problem is translation from one language to the other. The aim of these notes is to start this process and indicate that the translation might be possible.

On the strings side, the key words are something like:

* strings, branes

** extra dimensions

*** classical solutions.

We will argue that their counterparts on the lattice side are:

* low-dimensional defects $(1 \mathrm{~d}, 2 \mathrm{~d}, 3 \mathrm{~d})$

** spectrum of defects in their length, area

*** fine tuned field configurations which unify dependencies on both infrared and ultraviolet scales represented by $\Lambda_{Q C D}$ and inverse lattice spacing $a^{-1}$, respectively.

\footnotetext{
2 This viewpoint is not original of course, see, in particular, [6].

${ }^{3}$ Note, though, that the second paper in Ref [5] studies spectrum of gluons emitted and finds hard gluons, the result which can be trusted only for strings thin enough.
}

\section{Optimistic version of conclusions}

For the reader's orientation, let me mention at the very beginning that I am driving towards optimistic conclusions. I do feel that existing knowledge on confining fields on the lattice might fit the stringy picture in the continuum.

In particular, the center vortices could well be dual strings (or, actually, branes with one extra dimension compactified). The definition of the dual string is that it can be open on the 't Hooft line.

Lattice monopoles appear to be particles living on the dual strings. They could be an evidence for an extra dimension or, alternatively, for a crucial role of quantum fluctuations to stabilize the dual string in the infrared.

Topological fermionic modes could live on domain walls ( $3 \mathrm{~d}$ defects). The evidence in this case, however, is very preliminary.

Let me emphasize: I am putting the conclusions baldly without much reservations. It is not because the evidence is so solid but just to fix attention on the points which seem crucial. All interpretations are very tentative in fact.

\section{E. Material}

It would be logical to organize the material in the following way:

I Vacuum defects in Abelian cases, compact U(1) and $Z_{2}$ theories. Physics is well understood here since long. We need terminology, images to be used in the Yang-Mills case later.

II Vacuum defects in Yang-Mills case, lattice phenomenology and continuum-theory interpretation:

* $1 \mathrm{~d}$ defects - monopoles

$* * 2 \mathrm{~d}$ defects - center vortices

$* * * 3 \mathrm{~d}$ defects - volumes spanned on the vortices

$* * * * 3 \mathrm{~d}$ defects - space regions of topologically non-trivial gluon fields

III The string picture and traditional phenomenology $(<$ $A_{\min }^{2}>$, renormalons).

Because of space limitations we actually cannot cover the last topic in any systematic way and confine ourselves to a few remarks in the conclusions.

\section{DUAL-SUPERCONDUCTOR MODEL OF CONFINEMENT}

\section{A. Classical vs quantum}

One of central themes which goes through our discussion is the relation between classical solutions and quantum fields in vacuum. From theoretical point of view, it is quite a trivial point. However, it is important to appreciate this point to avoid confusion in discussing lattice data later.

(a) Already the Coulomb law is taught in physics courses in two different ways. First, we learn that classically we solve 
equation for the potential $\phi$ created by charge $e_{1}$

$$
\Delta \phi=e_{1} \rho
$$

and then evaluate the energy of another charge, $e_{2}$ in the potential created by the first charge. We arrive of course at

$$
V(r)=-\operatorname{const} \frac{e_{1} e_{2}}{r}
$$

Note that classically we start from 'empty' vacuum.

(b) Quantum mechanically, we evaluate the same potential energy as a result of interaction of the charges with zero-point fluctuations of the vacuum. These, vacuum fields are crucial for the derivation.

It is this, QM version which goes onto the lattice. Namely, one starts with the action

$$
S=\frac{1}{4 e^{2}} \int F_{\mu v}^{2} d^{4} x
$$

and generates vacuum field configurations $\left\{A_{\mu}^{v a c}\right\}$. These vacuum configurations do not know anything about external charges.

As a next step, one evaluates the Wilson line

$$
\left\langle\exp \left(i \int_{C} A_{\mu}^{v a c} d x_{\mu}\right)\right\rangle=\exp (-V(r) T)
$$

where the averaging is over the vacuum field configurations. The outcome is of course the same Coulomb potential, as derived classically.

Thus, we can say that zero-point fluctuations represent quantum, vacuum fields which correspond to the classical solution for the electrostatic potential.

(c) Our aim can be now formulated as follows. Imagine that the Wilson line in the YM case is given by a classical string solution in a $5 \mathrm{~d}$ curved space. We would like to determine then quantum, vacuum fields which, being substituted into the Wilson line and averaged over the configurations, reproduce the same result as the classical solution. To reiterate: on the lattice, we are not trying to detect directly the strings which can be open on the Wilson line. Instead, we can hope to detect/clarify vacuum fields (generated with the standard action $S=1 / 4 g^{2} \int G^{2} d^{4} x$ ) which correspond to classical string solution in extra dimensions.

\section{B. Abrikosov-Nielsen-Olesen vortex}

One starts with action of a charged scalar field interacting with electromagnetic field:

$$
S=\int d^{4} x\left(\frac{1}{4 e^{2}} F_{\mu v}^{2}+\left|D_{\mu} \phi\right|^{2}+V(\phi)\right)
$$

where the potential ensures a non-zero vacuum expectation value $v$ :

$$
V(\phi)=\left(|\phi|^{2}-v^{2}\right)^{2}
$$

One looks, furthermore, for a static solution with axial symmetry:

$$
\phi=\phi(\rho) e^{i \theta}, A_{\theta}=a(\rho)
$$

Contribution to the energy from large $\rho$ is finite only if:

$$
\phi(\infty)=v, a(\infty)=\frac{1}{\rho} .
$$

Eq. (8) alone allows to calculate the magnetic flux transported along the axis:

$$
\int_{0}^{2 \pi} d \theta A_{\theta} \cdot \rho=2 \pi
$$

Thus, the flux is quantized.

\section{Confinement dogma}

Because of the flux quantization, the vortex can end on magnetic monopoles. The vortex costs a finite energy per unit length and, therefore, the potential between the monopole and anti-monopole rises linearly at large distances:

$$
\lim _{r \rightarrow \infty} V_{M \bar{M}}=\sigma_{\text {Abrikosov }} \cdot r
$$

where the dimensionality for the string tension, $\sigma_{\text {Abrikosov }} \sim$ $v^{2}$, is provided by the Higgs particle condensate.

Interchanging electric and magnetic charges one gets the dual-superconductor model of confinement:

$$
\lim _{r \rightarrow \infty} V_{Q \bar{Q}}=\sigma_{\text {Abrikosov }} \cdot r
$$

where $Q$ is a heavy quark

$$
\sigma_{\text {Abrikosov }} \sim v_{M}^{2}
$$

and $v_{M}$ is a condensate of magnetically charge field, whatever it means.

The model introduces one of the basic concepts of all the confinement models:

condensation of electric charges implies confinement of magnetic charges

and, vice verse,

condensation of magnetic degrees of freedom implies confinement of color

The whole issue is now, what 'magnetic degrees of freedom' are. The version of answer which we will substantiate later is that magnetic degrees of freedom in the Euclidean vacuum are $2 \mathrm{~d}$ dimensional surfaces carrying magnetic flux (no quantization condition known) populated with particles. In the lattice-community terminology the $2 \mathrm{~d}$ surfaces are center vortices (for review see [7]) while the particles are monopoles (for review see [8]) 


\section{Quantum version of the dual superconductor}

Now, that we learned another classical solution, we can address our central question, what are quantum, vacuum fields which would reproduce the potential (11).

The answer to this question in the Abelian case is known since 1974 [9] and introduces new notions which are not necessarily taught at Universities. Namely, one visualizes vacuum in the following way. There are trajectories of (magnetically) charged particles in the vacuum. The trajectories are infinitely thin and closed, as result of (magnetic) charge conservation. The trajectories form clusters and there are two different types of clusters, finite and infinite. The infinite cluster is in a single copy for each configuration (for large but finite lattice it stretches from one boundary to another).

To get

$$
<W>\sim \exp \left(-\sigma_{\text {Abrikosov }} r \cdot T\right)
$$

one is to compute the gauge field $A_{\mu}$ created by particles belonging to the infinite cluster and substitute it (configuration after configuration) into the Wilson line. Moreover, the infinite cluster is very dilute.

Thus, there are a few ingredients to this picture which need to be explained:

* trajectories of virtual, magnetically charged particles,

* finite clusters, corresponding to quantum fluctuations of a charged field, and responsible for $<\left|\phi_{M}\right|^{2}>\neq 0$

$*$ infinite cluster of trajectories as representing nonvanishing classical field, $\left\langle\phi_{M}\right\rangle \neq 0$.

\section{E. Polymer representation of field theory}

The notion of trajectories of particles arises within the so called polymer approach to field theory, see. e.g., [10].

One starts with classical action of particle of mass $M$,

$$
S=M \cdot L,
$$

where $L$ is the length of trajectory. Next, introduce the path integral for the propagator:

$$
D\left(x, x^{\prime}\right) \equiv(\text { const }) \Sigma_{\text {paths }} \exp \left(-S_{c l}(\text { path })\right) .
$$

To enumerate all the paths one needs lattice. Note that lattice is needed here for pure theoretical reasons, not for computing

Usually one introduces (hyper)cubic lattice. Then the sum (15) can be made exactly. The result is that, indeed, $D\left(x, x^{\prime}\right)$ is proportional to the free field propagator. However, the classical mass parameter $M$ is not the propagating, physical mass. Instead, the propagating mass is given by

$$
m_{\text {prop }}^{2}=\frac{\text { const }}{a}\left(M(a)-\frac{\text { " } \ln 7^{\prime \prime}}{a}\right),
$$

\footnotetext{
${ }^{4}$ It is crucial that we consider Euclidean space-time. In Minkowski space, evaluation of the relativistic propagator is not a one-particle problem.
}

where " $\ln 7^{\prime \prime} \approx \ln 7^{5}$ and $a$ is the lattice spacing and we reserved for dependence of the mass parameter in (14) on the UV cut off.

A few comments concerning (16) are now in order.

First, Eq (16) can be viewed as a realization of a general relation:

$$
(\text { free energy })=(\text { energy })-(\text { entropy }),
$$

where "entropy" corresponds to the number of different trajectories of the same length $L$ (this number, obviously, appears in the process of evaluating the sum (15)). Indeed from a given point the trajectory can be continued in 7 directions, adding the same piece $a$ to the total length of trajectory. Here, the number ' 7 ' is related to the dimension of the space:

$$
7=4 d-1,(d=4) .
$$

Note that the backtracking is not allowed since we would just cancel the preceding step by going back.

Second, note analogy to the standard expression for the radiative correction to the Higgs mass:

$$
m_{\text {Higgs }}^{2}=\alpha \Lambda_{U V}^{2}-M_{0}^{2},
$$

where $\Lambda_{U V}$ is the UV cut off, $\alpha$ is the coupling and $M_{0}^{2}$ is a counterterm.

Similarity between (18) and (16) is that in both cases we need fine tuning for the physical mass. Namely, only if the classical mass of (14) is replaced by

$$
M(a)=\frac{{ }^{\prime} \ln 7^{\prime \prime}}{a}\left(1+O\left(m^{2} a^{2}\right)\right)
$$

do we have an interesting case. The peculiarity of (16) is that the subtraction constant $M_{0}^{2}$ is calculable in terms of entropy. The notion of fine tuning which appeared first in Higgs physics will be one of central for our course as well.

\section{Clusters}

For a positive propagating mass (16) one can find spectrum of finite clusters as function of their length $L$ [10]:

$$
N(L)=\frac{\text { const }}{L^{3}} \exp \left(-m_{\text {prop }}^{2} a L\right) .
$$

Eq (20) is an expression for the simplest vacuum loop in the polymer representation.

Eq (20) indicates that at $m_{\text {prop }}^{2}=0$ there is no exponential suppression for large lengths. Indeed, there is a phase transition at this point. Within the formalism we are considering now, the phase transition is manifested in appearance of an infinite, or percolating cluster. In the standard language the phase transition to Higgs condensation occurs at $m_{\text {Higgs }}^{2}=0$. Thus we identify the two masses:

$$
m_{\text {prop }}^{2}=m_{\text {Higgs }}^{2} .
$$

\footnotetext{
5 The approximation is to neglect other clusters. Numerically, it works within five per cent.
} 
In the terminology of percolation theory one can distinguish three regions:

$$
\begin{gathered}
m_{\text {prop }}^{2}<-- \text { subcritical phase } \\
m_{\text {prop }}^{2}=0-- \text { critical point } \\
m_{\text {prop }}^{2}<0-- \text { supercritical phase. }
\end{gathered}
$$

By far the least trivial is the theory of the supercritical phase.

Supercritical phase

The supercritical phase corresponds to a tachyonic mass, $m_{\text {prop }}^{2}<0$. Tachyonic mass signals instability of the original system and the new equilibrium position should be found. Generally speaking, it is not related to the original minimum of the action. In percolation theory, one considers the case when the transition is smooth ${ }^{6}$. In particular, the percolating cluster is very dilute near the point of the phase transition:

$$
L_{\text {perc }}=\frac{\text { const }}{a} V_{\text {tot }}\left(\left|m_{\text {prop }}^{2}\right| \cdot a^{2}\right)^{\alpha},
$$

the critical exponent $\alpha$ being positive, $\alpha>0$. Eq (21) is equivalent to the statement that the probability of a given link on the lattice to belong to the percolating cluster vanishes at the point of the phase transition:

$$
\theta_{\text {link }} \sim\left(\left|m_{\text {prop }}^{2}\right| \cdot a^{2}\right)^{\alpha} .
$$

Moreover, stabilization of the length of the infinite cluster can be described dynamically [11]. Consider the total length of the percolating cluster as an effective degree of freedom. Then one can suggest the following effective action for this degree of freedom:

$$
S_{\text {eff }}=-\varepsilon L_{\text {infinite }}+\frac{L_{\text {infinite }}^{2} a^{2}}{V_{\text {tot }}\left(\left|m_{\text {prop }}^{2}\right| a^{2}\right)^{\gamma}},
$$

where

$$
M(\varepsilon)=\frac{\ln 7}{a}-\frac{|\varepsilon|}{a} \quad(\varepsilon>0),
$$

and $\gamma$ is a new critical exponent, $\gamma>0$.

Let us comment on (23). The negative sign of the first term in the right-hand side corresponds to the tachyonic nature of the mode. It is stabilized by a term inverse proportional to the total volume $V_{\text {tot }}$. In the thermodynamic limit $V_{\text {tot }} \rightarrow \infty$. However, (23) is sufficient to ensure (21).

What might be most interesting about (23) is that it also predicts [11] fluctuations $\delta L$ of the length of the percolating cluster in the finite total volume:

$$
(\delta L)^{2} \sim V_{\text {tot }} a^{-2}\left(\left|m_{\text {prop }}^{2}\right| a^{2}\right)^{\gamma},
$$

and this relation can be checked independently.

\footnotetext{
6 The assumption on the smoothness of the phase transition constrains spectrum of finite clusters in subcritical phase.
}

\section{F. Monopoles in $\mathrm{U}(\mathbf{1})$ case}

General percolation relations, discussed so far, are realized within compact $U(1)$ theory (for a classical review see [12], for a recent review and further references see, e.g., [13]). The action is that of free photon field,

$$
S=\frac{1}{4 e^{2}} \int d^{4} x F_{\mu v}^{2}
$$

supplemented, however, with the condition that

\section{Dirac string costs no action}

which turns to be crucial for the dynamics of the model. On the lattice, the condition (26) is incorporated in the most natural way since one starts with the action written as

$$
S \sim \operatorname{Re}\left(\exp ^{\left(i \int_{\text {plaquette }} A_{\mu} d x_{\mu}\right)}\right)
$$

and the integral is indeed not sensitive to the Dirac string which gives a phase factor $2 \pi$.

Turn now to the dynamics of (25), with specification (26). Apart from trivial part, free photons, there is a contribution to the partition function from solitons, which are nothing else but magnetic monopoles. In the classical approximation, the mass of the monopole is given by

$$
M_{\text {monopole }}=\frac{1}{8 \pi} \int_{a}^{\infty} \mathbf{H}^{2}(r) d^{3} r
$$

where the radial magnetic field is

$$
\mathbf{H} \sim \frac{\mathbf{r}}{r^{3}} Q_{M},
$$

and the magnetic charge $Q_{M} \sim 1 / e$. The monopole mass (28) is UV divergent,

$$
M_{\text {monopole }} \sim \frac{\text { const }}{e^{2} a},
$$

where the constant is calculable explicitly on the lattice.

\section{G. Mapping to percolation}

Since the mass of the monopole is UV divergent, see (28), it seems reasonable to keep only self-energy and neglect interaction of the monopoles. The monopole action reduces then to (14), with the mass parameter $M(a) \sim 1 / a$. Thus, compact $U(1)$ theory reduces to the percolation theory ${ }^{7}$ and one

\footnotetext{
${ }^{7}$ Note that for small clusters the entropy is not realized in full, because of the condition of closeness of trajectories. As a result the phase transition is weak first order. The transition becomes of second order if one modifies in a certain way action for small clusters. We mention these points only in passing since we are not interested much in the Abelian examples by themselves.
} 
concludes that there is a phase transition [9], [14] at a critical value of the electric charge (which controls the coefficient in front of the UV divergence in the monopole mass). This critical value is given approximately by

$$
\frac{\text { const }}{e_{\text {crit }}^{2}} \approx \ln 7
$$

where the constant is the same as in $(28) e_{\text {crit }}^{2} \approx 1$ in case of the cubic lattice.

Another crucial point for the mapping to percolation is that the monopole trajectories can be defined in terms of violations of the Bianchi identities:

$$
\partial_{\mu} \tilde{F}_{\mu v} \equiv j_{v}^{m a g n} .
$$

Let us pause here to emphasize that the definition of the monopole current is highly non-trivial. First, one needs a lattice version of the Bianchi identities, and it indeed exists [15]. Second, it is very important that the definition (30) is valid on a configuration level. Indeed, say, equations of motion are not valid on a configuration level. They are valid only on average, according to Ehrenfest:

$$
\left\langle\partial_{\mu} F_{\mu \nu}\right\rangle=0
$$

while for any particular configuration $\partial_{\mu} F_{\mu v}$ is not vanishing at all. The possibility to introduce the definition (30) on the configuration level is due to the fact that the Bianchi identities have kinematical nature. It goes without saying that violations of the Bianchi identities, (30) assume singular fields.

\section{H. Summary on the compact $\mathrm{U}(1)$}

a Confinement is well understood both classically and QM in terms of condensation of dual charges.

b For us, the fine tuning is the central point. Namely, the mass is represented as

$$
m_{\text {phys }}^{2}=\frac{\text { const }}{a}\left(\frac{\text { const }}{a}-\frac{\ln 7}{a}\right),
$$

and the coupling $e^{2}$ is to be very close to its critical value, $e_{c r i t}^{2}$, for the mass to be in units of $1 / a \varepsilon, \varepsilon \equiv e^{2}-e_{c r i t}^{2} \ll 1$. The classical vacuum field is then:

$$
<\phi_{M}>\sim \frac{1}{a} \varepsilon^{\delta}, \delta>0,
$$

and also vanishes in the limit $\varepsilon \rightarrow 0$.

c The fine tuning implies that the free energy does not depend on the lattice spacing. Many observables can be discussed directly in terms of the free energy, or physical mass (32) and do not exhibit then the fine tuning. Measuring the action (or entropy) separately and observing the fine tuning implies zero (in the limit $a \rightarrow 0$ ) size of the monopoles. Indeed, if the monopole has, say, a Higgs core, the action is no longer divergent inside the core. The action is divergent only as far as the monopole is point-like.

In this sense, observing fine tuning in the Euclidean space is similar to observation of point-like particles in DIS in the Minkowski space.

\section{CONFINEMENT IN $Z_{2}$ GAUGE THEORY}

\section{A. Formulation of the theory}

One of central points of dual formulations of YM theories is an expression for the Wilson line in terms of surfaces spanned on the Wison contour $C$ :

$$
\langle W\rangle=\operatorname{const} \Sigma_{A_{C}} \exp \left(-\ldots A_{C}\right),
$$

where $A_{C}$ is the area of the surface and derivation of the weight function, denoted by dots is in fact a central issue for the theory.

Historically, an explicit expression of the type (34) was derived first in case of the $Z_{2}$ gauge theory (for review see, e.g., [16]). The partition function of the model is given by integral over all links which take on values

$$
\text { links } l_{n, \mu}= \pm 1
$$

while the action depends on plaquette values. The plaquette is given by the product of the four links,

$$
(\text { plaquette })=\prod_{i=1}^{i=4} l_{i},
$$

and takes on values \pm 1 , depending on the links. Finally, the action is

$$
S=\beta A_{-},
$$

wher $A_{-}$is the total area of all the negative plaquettes and $\beta$ is a constant.

\section{B. Center vortices}

Instead of a negative plaquette one can introduce a plaquette orthogonal to it and belonging to the dual lattice. Note that the two plaquettes intersect in $4 \mathrm{~d}$ at a single point (which is the middle of the plaquettes).

Collection of all such plaquettes on the dual lattice is called center vortex, or P-vortex. The advantage of considering the center vortices is that they are closed by construction and in this sense topological.

Next, one can consider clusters of center vortices, similar to the monopole case considered above. Infinite, or pecolating cluster of center vortices is central for the confinement theory in the $Z_{2}$ case.

\section{C. $Z_{2}$ duality}

Geometrically, it is quite obvious that $Z_{2}$ theory on the dual lattice is a $Z_{2}$ theory again. In other words, the theory is self dual. In more detail, (for review see, e.g., [16]) the dual coupling is given by

$$
\beta^{*}=-\ln \tanh \frac{\beta}{2}
$$


where the coupling $\beta$ is introduced in Eq (37). Note that if $\beta \rightarrow 0$, the dual coupling tends to infinity, $\beta^{*} \rightarrow|\ln \beta|$. And vice verse, if $\beta \rightarrow \infty, \beta^{*} \rightarrow 2 \exp ^{-\beta / 2} \rightarrow 0$. The meaning of self duality is that the average values of the plaquette $E(\beta)$ and $E\left(\beta^{*}\right)$ are related:

$$
E\left(\frac{\beta}{2}\right)=1-\tanh \frac{\beta}{2}-\left(\sinh \frac{\beta}{2}\right) E\left(\frac{\beta^{*}}{2}\right) .
$$

The self-dual point, $E(\beta)=E\left(\beta^{*}\right)$, is at

$$
\beta=\beta_{c r}=2 \ln (1+\sqrt{2}) \approx 0.88 \text {. }
$$

The Wilson line is defined as product of all the links along the contour $\mathrm{C}$ and one can prove:

$$
\langle W(C)\rangle=(\text { const }) \Sigma_{A_{C}} \exp \left(-\beta^{*} A_{C}\right),
$$

where $A_{C}$ is area of a surface spanned on the Wilson contour $C$. Note the dual coupling (38) as the weight factor.

In particular, if $\beta \rightarrow 0$ then $\beta^{*} \rightarrow \infty$ and the sum (41) is dominated by the smallest area surface. We have, therefore, the area law and the string tension $\sigma \approx a^{-2} \beta^{*}$.

\section{D. 't Hooft loop}

The next question is what is relation of the confinement we have just derived to condensation of dual degrees of freedom.

To answer this question consider the 't Hooft line, which in case of the $Z_{2}$ is nothing else but the Wilson line on the dual lattice. In more detail, let is introduce field of Dirac string, $A_{\mu}^{\text {Dir }}$ such that

$$
\exp \left(i \int_{\text {plaquette }} A_{\mu}^{\text {Dir }} d x_{\mu}\right)=-1
$$

where we for a moment use notations of continuum U(1) theory, not of $Z_{2}$ theory. The reason for using such notations is that the 't Hooft loop can be introduced in the U(1) and YM cases as well. Note that the Dirac string (42) is visible in the sense that it does cost action (unlike a more conventional Dirac string which gives the phase factor $2 \pi$ ).

Let the Dirac string (42) pierce a stack of negative plaquettes. Then the trajectory of the end points of the string is called 't Hooft loop. The end points of the Dirac string are magnetic monopoles. Moreover, the heavy-monopole potential $V_{M \bar{M}}$ is introduced now in terms of a rectangular 't Hooft loop.

The 't Hooft loop is a line on the dual lattice. It seems rather obvious that center vortices which are closed surfaces on the dual lattice can be open on the 't Hooft line. Indeed, a stack of negative plaquettes introduced to the vacuum as a 't Hooft line, completes then an open center vortex to a closed one.

With this insight, it is easy to accept that the 't Hooft loop can be evaluated as a sum over surfaces:

$$
\langle H\rangle \sim \Sigma_{A_{H}} \exp \left(-\beta A_{H}\right),
$$

where $\beta$ is the coupling of our original formulation, see (37). From selfduality of the model, by changing $\langle W\rangle$ to $\langle H\rangle$ and $\beta$ to $\beta^{*}$ one derives then Eq. (41) for the Wilson loop.
We come now to discuss a crucial point: both Wilson and 't Hooft lines have similar expressions in terms of area of surfaces spanned on the lines. On the other hand, it is either heavy quarks or heavy monopoles that are confined. Therefore, one of the representations (41), (43) is in fact formal. In the sense that the sum over surfaces diverges since the entropy of surfaces wins over the suppression due to the action. The suppression is explicit in (41) and (43) while the enhancement due to the entropy is implicit and is hidden in the symbol of summation over all the surfaces. The reason for an exponential enhancement due to entropy is similar to the case of trajectories, discussed in detail in the preceding section. Namely, plaquettes belonging to center vortices can be continued in a few directions preserving the same total area.

Thus, one can visualize the transition from confinement to deconfinement in the following way. Start with small $\beta$ and, therefore, large $\beta^{*}$. Then according to (41) there is area law for the Wilson line, or confinement. Increasing $\beta$ and decreasing $\beta^{*}$ weakens dominance of the minimal area in the sum (41). The string tension goes down. In this region the contribution of large-area surfaces in (41) increases. The role of the constraint that the surfaces is bound by the Wilson contour is becoming less and less important since the surfaces have larger and larger area. Finally, infinite-area surfaces become allowed. There appears an infinite percolating cluster on the direct lattice and the confinement is lost.

With evaluation of the 't Hooft line, the logic is the same, with interchange of $\beta$ and $\beta^{*}$. If there is a single phase transition then, from symmetry consideration it occurs at the selfdual point (40). At this point the area law for the Wilson line is interchanged into the area law for the 't Hooft line.

\section{E. Stochastic model}

Consideration of the preceding subsection establishes relation between existence of a percolating cluster of center vortices on the dual lattice and area law for the Wilson line on the direct lattice. Stochastic model makes this relation more quantitative (for a detailed review see [17]; below we follow closely [7]).

First, let us rewrite the expression for the Wilson loop:

$$
\langle W\rangle=\left\langle\Pi_{C} l_{i}\right\rangle \equiv\left\langle\Pi_{A_{C}}(\text { plaquettes })\right\rangle .
$$

In other words, we replace the product of links covering the Wilson contour $\mathrm{C}$ by product of plaquettes covering the area $A_{C}$ of a surface spanned on the contour $C$. Completion of the product of the links to the product of plaquettes adds product over extra links and each of these links enters twice. Since $l_{n, \mu}^{2}=+1$ rewriting (44) is an identity.

Plaquettes on the original lattice are negative if they are pierced by a center vortex (by definition). Therefore,

$$
\langle W\rangle=\left\langle(-1)^{I}\right\rangle,
$$

where $I$ is the number of intersections of the surface $A_{C}$ with center vortices.

Note furthermore that finite clusters of center vortices cannot give the area law. The reason is that for a large enough 
contour $\mathrm{C}$, finite clusters intersect the surface $A_{C}$ twice and give a trivial factor to (45). The argument does not hold for finite clusters which are close to the boundary of the surface, or contour $C$. Indeed, the second intersection can then happen outside the surface $A_{C}$ and contribution to (45) survives. However, the condition of closeness of the finite clusters to the boundary means that they can produce only the perimeter law for the Wilson line, not the area law.

Now, the stochastic model is the assumption that the probability for a given plaquette to be pierced by a center vortex is independent of other plaquettes. Then:

$$
\langle W\rangle=\langle\Pi(\text { plaquettes })\rangle \approx \Pi\langle(\text { plaquette })\rangle .
$$

Moreover, for the average value of the plaquette we have:

$$
\langle(\text { plaquette })\rangle=(-1) \cdot p+(+1) \cdot(1-p),
$$

where $p$ is the probability of a given plaquette to be pierced by the infinite, percolating cluster of center vortices.

Collecting all these simple equations and assuming that we can choose minimal surface for $A_{C}$ in Eq (44) we get

$$
\langle W\rangle \approx \exp \left(-2 p A_{\min }\right)
$$

where $p$ is the probability introduced above. Note that there is one extra assumption: choosing the minimal surface. It could be substantiated to some extent but not actually derived.

In conclusion of this subsection let us emphasize importance of the stochastic model. The point is that studying vacuum state does not reveal directly surfaces which can be open on the Wilson line and provide the area law. Instead, one can observe condensation of the dual degrees of freedom, which are center vortices living on the dual lattice in our $Z_{2}$ case. Stochastic model allows to estimate the confining string tension in terms of the vacuum fields.

\section{F. Branched polymers}

Following the U(1) suit, we would like to have fine tuning:

$$
\left(\text { Tension } \sim \varepsilon / a^{2}\right)=\left(\text { Action } \sim 1 / a^{2}\right)-\left(\text { Entropy } \sim 1 / a^{2}\right),
$$

with $\varepsilon \ll 1$. The entropy was measured numerically only very recently [18]:

$$
N_{\text {area }} \approx \exp \left(+c_{K} \frac{\text { Area }}{a^{2}}\right), c_{K} \approx 0.86
$$

where $N_{\text {area }}$ is the number of various surfaces with the same area. Note that $c_{K}$ is close to but different from the self-dual point (40).

Thus, one could expect that fine tuning is easy to achieve. On the other hand, if it were indeed so, then we would have consistent theory of strings in $4 \mathrm{~d}$, which is in contradiction with well known results. The resolution of the paradox is that the fine tuned surfaces are very specific. They are what is called branched polymers $[10,18,19]$. The branched polymers are very thin tubes so that the $3 \mathrm{~d}$ volume bound by the surface is approximately

$$
V_{3 d} \approx \frac{\text { Area }}{4} \cdot a
$$

Since the branched polymers are in fact trajectory-like they correspond to theory of a real scalar field.

\section{G. Summary on $Z_{2}$}

Both Wilson and 't Hooft loops are given by sums over surfaces spanned on the corresponding contours:

$$
\langle W\rangle \sim \Sigma_{A_{C}} \exp \left(-\beta^{*} A_{C}\right), \quad\langle H\rangle \sim \Sigma_{A_{H}} \exp \left(-\beta A_{H}\right)
$$

where $A_{C}, A_{H}$ are the areas of the surfaces.

However, only one of the sums signals the area law. Namely, that one which involves larger coupling, $\beta$ or $\beta^{*}$. The other sum diverges because the (implicit) entropy factor prevails. If, say, $\beta^{*}>\beta$, this divergence implies existence of an infinite cluster of surfaces on the dual lattice. This cluster is responsible for confinement on the direct lattice. Quantitatively, the string tension can be estimated by invoking the stochastic model.

\section{FINE TUNING VS ASYMPTOTIC FREEDOM}

\section{A. Fine tuning}

Let us emphasize again one of central messages of the lectures: fine tuning between entropy and action is a signature of an elementary object, let it be particle or string. For particles, the polymer approach to field theory (in Euclidean spacetime) makes everything explicit. For strings, the fine tuning is rather a goal [10] than realization because there are no known consistent string theories in $4 \mathrm{~d}$. Technically, fine tuning for strings ( $2 \mathrm{~d}$ surfaces in Euclidean space) with the Nambu-Goto action fails because the surfaces decay into branched polymers which is another image of a real scalar field [10].

Thus, once we start looking for fundamental strings we are looking for fine tuned surfaces, with infinite action and entropy. Is it reasonable at all? The problem is that monopoles of the compact U(1) considered above in detail are ordinary point-like particles. It is clear that such particles are not allowed in the non-Abelian case. Indeed because of the asymptotic freedom short distances are to be described in terms of gluons alone. In the U(1) case, on the other hand, monopoles could be tuned to be light at large coupling of order unit. Thus, fine tuning in non-Abelian case is apparently much more subtle than in compact $\mathrm{U}(1)$.

What does it mean, that we are not allowed to introduce new particles in more technical terms? We already emphasized a few times that it is the behavior in the ultraviolet which is constrained by the asymptotic freedom. We will accept the following conjecture: 
All the UV divergences in matrix elements of (gauge invariant) local operators are to be calculable in terms of gluons and their interactions perturbatively.

Note that the 'UV divergences' include now power-like divergences as well. In the continuum, one is frequently saying that the power-like divergences are ambiguous. But once we fixed the regularization to be the lattice regularization, we fixed power-like divergences as well.

\section{B. Constraints on particles}

Imagine that we somehow managed to identify trajectories of magnetic monopoles (now, in non-Abelian case) and can introduce then a magnetically charged field $\phi_{M}$. For an elementary field, the vacuum expectation value of $\left|\phi_{M}\right|^{2}$ is of order:

$$
\left\langle\left. 0|| \phi_{M}\right|^{2} \mid 0\right\rangle \sim \frac{\text { const }}{a^{2}} .
$$

Such a vacuum expectation value, (52) is not allowed in the Yang-Mills case because there is no gauge invariant quadratic divergence in terms of gluons. Obviously, what is allowed:

$$
\left\langle\left. 0|| \phi_{M}\right|^{2} \mid 0\right\rangle \sim \Lambda_{Q C D}^{2},
$$

since it does not involve any UV divergence.

Since we are working in the polymer formalism, our next step is to translate the constraint (53) into the language of clusters. Consider again for a moment an elementary field (52). Introduce total density of monopoles:

$$
\left\langle L_{\text {tot }}^{\text {mon }}\right\rangle \equiv 4 \rho_{\text {tot }}^{\text {mon }} \cdot V_{t o t} .
$$

Moreover, the total length of the monopole trajectories, $L_{t o t}^{\text {mon }}$ is obtained by differentiating the partition function with respect to the mass parameter $M$ introduced in the classical action (see section II). On the other hand, $\left\langle\left. 0|| \phi_{M}\right|^{2} \mid 0\right\rangle$ is obtained by differentiating the partition function with respect to the propagating, or physical mass $m_{\text {prop }}^{2}$. We have also derived an explicit relation between the initial mass parameter $M$ and the physical mass, $m_{\text {prop }}^{2}$. From this relation we find

$$
\left\langle\left. 0|| \phi_{M}\right|^{2} \mid 0\right\rangle=(\text { const }) \rho_{\text {tot }}^{\text {mon }} \cdot a,
$$

where $a$ is the lattice spacing.

Let us check (55) in case of elementary particle. Consider to this end clusters of small size (small on the scale of $m^{-1}$ ). Then, there is no mass parameter and therefore:

$$
\rho_{\text {tot }}^{\text {mon }} \sim \frac{\text { const }}{a^{3}} \equiv \frac{\text { const }}{a^{d-1}},
$$

where $d$ is dimension of space available ${ }^{8}$. Substituting (56) into (55) we, naturally, reproduce the quadratic divergence (52) associated with the fundamental scalar field.

\footnotetext{
${ }^{8}$ In percolation theory, one introduces probability $p$ of a given link to belong to a trajectory. Near the phase transition $p \approx 1 / 7$ and $\rho_{\text {mon }}^{t o t} \approx a^{-3} / 7$.
}

Note that it is the density of short clusters that dominates the v.e.v. (55). The percolating cluster is fine tuned, or very dilute near the point of phase transition. Its contribution to (55) is negligible (while its role for confinement is crucial).

Now, we see that the monopole density in the YM case allowed by (53) is of order

$$
\rho_{\text {tot }}^{\text {mon }} \sim \frac{\Lambda_{Q C D}^{2}}{a} \equiv \frac{\Lambda_{Q C D}^{2}}{a^{2-1}} .
$$

Thus, geometrically, the asymptotic-freedom constraint implies that the magnetically charged particles live on a $2 \mathrm{~d}$ subspace of the whole $4 \mathrm{~d}$ space [20].

It is amusing that asymptotic freedom alone brings us so close to considering $2 \mathrm{~d}$ defects, or strings.

\section{Asymptotic freedom and strings}

To have a consistent theory of fundamental strings means to realize the fine tuning. Namely, the action for infinitely thin, or fundamental strings is to be ultraviolet divergent

$$
S_{\text {string }} \sim \frac{\text { Area }}{a^{2}} \text { const }
$$

while the tension, or area is to be in physical units:

$$
(\text { Area })_{\text {strings }} \sim \frac{1}{(\text { tension })} \sim \Lambda_{Q C D}^{2} \cdot V_{\text {tot }}
$$

If such strings exist, their contribution to gluon condensate (plaquette action) is of order:

$$
\left\langle\left(G_{\mu \nu}^{a}\right)^{2}\right\rangle_{\text {strings }} \sim \frac{\Lambda_{Q C D}^{2}}{a^{2}},
$$

where we keep track only of powers of the UV and IR parameters $\left(1 / a\right.$ and $\Lambda_{Q C D}$, respectively). Estimate (60) can be compared to the contribution to the action of the zero-point fluctuations,

$$
\left.\left\langle\left(G_{\mu \nu}^{a}\right)^{2}\right)\right\rangle_{\text {gluons }} \sim a^{-4},
$$

and with contribution of quasiclassical fields, 'instantons':

$$
\left.\left\langle\left(G_{\mu \nu}^{a}\right)^{2}\right)\right\rangle_{\text {instantons }} \sim \Lambda_{Q C D}^{4}
$$

Thus, strings give a subleading UV divergence into the plaquette action. According to the conjecture we formulated above it should also be calculable in terms of gluons.

Terms of order $\Lambda_{Q C D}^{2} / a^{2}$ in the plaquette action have been discussed since long, for details and references see [21]. These terms correspond to the so called ultraviolet renormalon which is a perturbative series with factorially growing expansion coefficients. The series is, however, Borel summable. Moreover, basing on the asymptotic freedom one can demonstrate that the perturbative series is to be understood as such a sum [22].

In other words, the contribution of order (60) to the plaquette action is calculable perturbatively. Thus, one concludes 
that contribution of the strings into the plaquette action is dual to high orders of perturbation theory. Note that the instanton contribution (62) is a non-perturbative counterpart of the so called infrared renormalon which is not summable and there is no duality for instantons.

\section{DUAL STRINGS ON THE LATTICE}

\section{A. Where we are now}

We actually reviewed (rather superficially) topics from a few theoretical disciplines:

\section{Field theory}

Confinement, in general terms, is an instability of the perturbative vacuum. Color is conserved and therefore one ascribes instability to condensation of magnetic degrees of freedom. Moreover, there is no Higgs field and the only way to introduce magnetic degrees of freedom seems to allow for violations of Bianchi identities:

$$
D_{\mu} G_{\mu \nu}=0, \quad D_{\mu} \tilde{G}_{\mu \nu} \neq 0
$$

We did not start, however, from these equations because the only solution which we are aware of - magnetically charged particles, or monopoles- would not work in the non-Abelian case. Indeed, postulating non-vanishing magnetic current, $j_{\mu}^{a}$, would introduce new colored particles and, therefore, modify equations of motion as well.

Thus, we concentrated on the observation that violations of the Bianchi identities assume existence of singular fields. On the other hand, asymptotic freedom of Yang-Mills theories severely constrains types of singular fields. We argued that what is allowed are particles living on $2 \mathrm{~d}$ surfaces (i.e. on a submanifold of the whole $4 \mathrm{~d}$ space). With stretch of imagination we can assume that the actual object is closed magnetic strings, populated with particles. The closeness of the strings is needed to avoid introducing new colored objects as the end points. Of course, at this moment such a hypothesis looks pure speculation. But we shall see later that it is in fact supported by the lattice data.

\section{Quantum geometry}

Quantum geometry formulates field theory and string theory in Euclidean space as theories of trajectories and surfaces, respectively. The central point is that both particles and strings are described in terms of fine tuning.

We discussed also the notions of clusters, percolation and so on. In particular, the phase transition to condensation corresponds to emergence of an infinite, or percolating cluster. Note that quantum geometry introduces lattice. This is so to say theoretical lattice needed to regularize theory in the UV, not to necessarily computerize the problem.

\section{String theory}

Dual formulation of Yang-Mills theory can well be string theory, for review see [23]. We emphasize here only one point. Namely, the strings which can be observed as vacuum defects are not the strings which can be open on the Wilson line but rather dual strings, which can be open on the 't Hooft loop.
Thus, by 'magnetic degrees of freedom' we should understand then closed strings which can be open on the 't Hooft line. Such strings are commonly considered in string theories dual to Yang-Mills theories, for references see [1,23].

One of central points of theories with extra dimensions in the dual formulation of Yang-Mills theories is an expression for the Wilson line. Rather schematically:

$$
\langle W\rangle=\operatorname{Const} \Sigma_{A_{C}} \exp \left(-f\left(G_{\mu v}\right) A_{C}\right),
$$

where $f\left(G_{\mu \nu}\right)$ is a function of the metrics in extra dimensions, see an example (3). Eq (64) can be considered actually as a starting point of the string picture.

If Eq. (64) holds then it seems natural to assume that a similar expression is true for the 't Hooft loop as well:

$$
\langle H\rangle=\text { Const } \Sigma_{A_{H}} \exp \left(-\tilde{f}\left(G_{\mu v}\right) A_{H}\right),
$$

where $\tilde{f}\left(G_{\mu v}\right)$ is another function of the same metrics.

Generically, it is either color or magnetic charges that are confined [24]. Therefore, in Yang-Mills case, (65) is to be formal, i.e. not produce in fact the area law despite of the factor $A_{H}$. From the $Z_{2}$ example we know how a sum like (65) can be reconciled with the perimeter law:

* entropy of the surfaces prevails over the suppression due to the area $A_{H}$ factor in the exponent,

* large areas $A_{H}$ become then allowed by (65),

* percolating cluster of surfaces is formed in the vacuum.

\section{Conclusions}

We can expect existence in vacuum state of Yang-Mills theories of an infinite (percolating) cluster of closed strings which can be open on the 't Hooft loop.

The natural next step is to compare theoretical expectations with lattice data.

\section{B. Center vortices}

Let us reiterate definition of the vacuum defects to be looked after on the lattice:

Closed, infinitely thin surfaces which can be open on the 't Hooft line.

At first sight, the definition is not specific enough, and we actually do not know whether there are further points to be added to the definition. Note, however, that the definition we have already now is highly non-trivial.

First of all, it is formulated on the scale of lattice spacing, or in ultraviolet. In this sense the definition is similar to, say, definition of monopoles in the U(1) case,

$$
\partial_{\mu} \tilde{F}_{\mu \nu} \equiv j_{v}^{m o n}
$$

which fixes the monopoles uniquely. In the classical approximation the 't Hooft loop introduces a heavy-monopole pair with Abelian charges corresponding to the flux brought in by the Dirac string, for derivation see [25]. Thus, magnetic strings can be open on the line along which the Bianchi identities are violated, without violating color conservation.

It is amusing to learn that apparently the lattice phenomenology is rich enough to allow identification on the lattice 
strings satisfying the definition above. These are nothing else but the center vortices [26] which were studied in great detail, for review see [7]. The lattice definition of the vortices is quite complicated and is given in specific lattice terms. We will come back to this definition in the next subsection.

Now, let us quote results of measurements of basic characteristics of these surfaces.

\section{Total area}

First, one can measure the total area of the surfaces. Total area is controlled by the string tension. The main observation is that the total area scales in the physical units ${ }^{9}$ :

$$
A_{\text {tot }} \approx 4(f m)^{-2} V_{t o t}
$$

The same result can be reformulated as a statement that probability of a given plaquette to belong to the center vortices is proportional to:

$$
\theta_{\text {plaquete }} \sim\left(a \cdot \Lambda_{Q C D}\right)^{2} .
$$

Thus, the cluster becomes very dilute in the limit $a \rightarrow 0$ and we can suspect that the surfaces are fine tuned.

\section{Non-Abelian action}

The non-Abelian action associated with the strings can be measured directly and it turns to be ultraviolet divergent: [27]

$$
S_{\text {tot }} \approx 0.54 \frac{A_{t o t}}{a^{2}}
$$

where by the action associated with the surfaces one understands the difference between the average action on the plaquettes belonging to the surfaces and the average over the whole lattice.

\section{Alignment of surface and of non-Abelian field}

In the same Ref. [27] one finds results of measurement of the extra action associated with plaquettes next to those pierced by the center vortices. The result for this action is null.

In other words, the whole excess of the action is carried by infinitely thin surfaces. Moreover, from this measurement we learn not only that the surface is thin but also that the nonAbelian field is aligned with the surface.

Thus, center vortices represent 'magnetic sheets' with the non-Abelian field collimated along the surface. The surfaces are fine tuned: their area is in physical units while their nonAbelian action is ultraviolet divergent. The surfaces form infinite, or percolating cluster.

\section{Projected fields}

Technically, the center vortices in the YM case are defined in terms of so called projected fields which replace - according to a certain algorithm - original $S U(2)$ links by $Z_{2}$ links.

\footnotetext{
9 The number is borrowed from Ref [27] and has the smallest error bars. The very effect of scaling of the area was discovered earlier [26], for further references see [7].
}

The projection is performed in two steps. First, one maximizes traces of the matrices $U_{n, \mu}$ which are the original $S U(2)$ fields. To this end, one fixes the gauge in such a way that the sum over the whole of the lattice,

$$
F(U)=\Sigma_{n . \mu}\left(\operatorname{Tr} U_{n, \mu}\right)^{2}
$$

takes on its maximum value. In other words, the links are gauge rotated as close as possible to the elements of the center group, which are $\pm I$.

Note that fixing this gauge, $\bar{U}_{n, \mu}$ does not modify the system at all. Crucial is the next step when one replaces the original links by $Z_{2}$ links:

$$
\bar{U}_{n, \mu} \rightarrow \bar{Z}_{n, \mu} \text {, where } \bar{Z}_{n, \mu} \equiv \operatorname{sign}\left(\operatorname{Tr} \bar{U}_{n, \mu}\right) \text {. }
$$

Finally, the center vortices are defined in the same way as in case of $Z_{2}$ theory, but this time in terms of the projected fields (70).

The projection and the corresponding center vortices are uniquely determined. However, one could choose another $Z_{2}$ projection and introduce corresponding vortices. In fact, there are infinitely many different $Z_{2}$ projections of non-Abelian fields. In particular, one can find such projections that the corresponding center vortices do not at all have properties like (66), (68) quoted above for measurements in the maximal center projections.

A phenomenological answer to this kind of concerns and criticism was given in Ref [28]. Namely, there was found a criterion which allows to distinguish between acceptable and unacceptable projections. One introduces by hand negative non-Abelian plaquettes on the lattice which form a closed surface. If projection allows to find this implanted thin vortex then the corresponding center vortices turn to possess properties similar to (66) and (68). The implanted closed thin vortex can be open on the 't Hooft line (which is end-line of a surface consisting of negative non-Abelian plaquettes). Then it is only natural to assume that other closed surfaces in the vacuum found by the same algorithm can also be open on the 't Hooft line. Thus, the phenomenological criterion discovered in Ref [28] gets justified on theoretical grounds.

\section{Strings explaining phenomelogy}

Let us dwell on this point somewhat longer. The centervortex model of confinement is a success, without any reference to (fundamental) strings. However, originally [26] the model was motivated by the idea of $Z_{2}$ dominance: one replaces original fields by $Z_{2}$ degrees of freedom and still reproduces confinement.

It is indeed known since long that the center of the group is relevant to the confinement [29]. However, the standard picture is that on the plaquette level one can forget about the center group and the knowledge on the center group is accumulated only after many steps $n_{\text {link }}$ along the Wilson line,

$$
n_{\text {link }} \sim\left(\Lambda_{Q C D} \cdot a\right)^{-1}
$$


The reason is that the continuum limit in asymptotically free theories is defined as an expansion near the unit matrix,

$$
U_{n, \mu} \approx I+i A_{n, \mu}^{a} \lambda^{a} / 2
$$

where $\lambda^{a} / 2$ are generators of the group and $A_{n, \mu} \sim g(a) / a$ standard continuum-theory gauge fields. Thus, the second element of the center of the group , matrix $-I$, is infinitely far from the continuum on the plaquette level. That is why finding success of the $Z_{2}$ projections on the plaquette level was a real breakthrough in studies of confinement ${ }^{10}$.

Now, we see that the center vortices are very natural if one thinks in terms of fundamental strings. Thus, stringy picture suggests the first ever explanation why phenomenology of the thin center vortices turns so successful. The "finding property' of $Z_{2}$ projections [28] links the phenomenological center vortices to fundamental dual strings.

\section{E. Three-dimensional defects}

As we discussed in section IIIF, the only theoretically known example of fine-tuned surfaces are branched polymers, which in fact correspond to a scalar particle. Thus, it is extremely importnat to check whether the lattice strings are branched polymers ot not. For branched polymers the minimal $3 \mathrm{~d}$ volume bound by the surface is approximately $V_{3 d} \approx \frac{a}{4}($ Area $)$, where $a$ is the lattice spacing.

In case of SU(2) Yang-Mills theory the minimal volume was measured in [31] and found to scale in physical units:

$$
V_{3 d} \approx 2 \mathrm{fm}^{-1} V_{t o t} \approx \frac{1}{12} f m(\text { Area })
$$

where (Area) now stands for the total area of the $2 \mathrm{~d}$ defects.

As far as the $3 \mathrm{~d}$ volume scales in physical units, as is indicated by (73) the $2 \mathrm{~d}$ defects are not branched polymers. It is an important conclusion. In view of importance of the scaling law (73) its further checks seem well justified.

Measurements (73) establish existence of new defects, three-dimensional volumes. Their relevance to confinement and spontaneous breaking of chiral symmetry is revealed by procedure which is commonly called removal of center vortices [30]. To remove the center vortices from the lattice, configuration after configuration it was suggested [30] to modify the original fields in the following way:

$$
U_{n, \mu} \rightarrow \tilde{U}_{n, \mu} \text { where } \tilde{U}_{n \mu}=\bar{Z}_{n, \mu} U_{n, \mu},
$$

and $\bar{Z}_{n, \mu}$ are the $Z_{2}$ projections of the links (see (70)). It was found that confinement is lost after the modification (74).

The actual question is how 'massive' is the ad hoc change (74). As far as the plaquettes are concerned the modification

\footnotetext{
${ }^{10}$ To reconcile this success with the standard picture (71) one postulates that infinitely thin vortices are in one-to-one correspondence with so called thick vortices which are hypothetical bulky fields with size of order $\Lambda_{O C D}^{-1}$ [7]. However, such an assumption is difficult to justify theoretically.
}

(74) affects only plaquettes pierced by the center vortices, and such plaquettes are rare, see (66). In quantum mechanics, however, the gauge potential is physically meaningful and modifying potential even without modifying the corresponding field strength tensor can change physics drastically. The original measurements [30] were made in the gauge where approximately one half of the projected links took value $(-1)$. Thus, the ad hoc modification (74) affected half of the lattice and and the loss of confinement might look not so surprising.

A more refined way is to introduce such $Z_{2}$ gauge which minimizes the number of negative projections [31]. It is straightforward to realize that in this way one comes to the same three-dimensional volumes introduced above. Thus the volume (73) constitutes the minimal volume which - in a certain gauge - carries information both on confinement and chiral symmetry breaking. Let us emphasized that these $3 \mathrm{~d}$ volumes occupy a fraction of the total volume which vanishes in the continuum limit:

$$
\theta_{3 d} \sim\left(a \cdot \Lambda_{Q C D}\right)
$$

where $\theta_{3 d}$ is the probability of a given lattice cube to belong to the minimal volume.

Thus, we come to a kind of holographic principle: using gauge invariance one can encode the whole information on the confinement on a submanifold of the $4 \mathrm{~d}$ space, percolating through the total volume.

\section{F. Singular stochastic fields}

We already introduced the stochastic model in connection with the $Z_{2}$ gauge theory. The stochastic component of vacuum fields in that case was provided by percolating cluster of center vortices. Now, that we have learned that in the YM case there also exists a percolating cluster of magnetic vortices, it is natural to apply the stochastic model to evaluate the Wilson line as well. Generally speaking, derivation of the stochastic model in the non-Abelian case is much more complicated. We will see that, amusingly enough, the singular nature of the non-Abelian fields associated with the dual strings makes application of the stochastic model much more straightforward.

Let us reiterate the basic steps of the stochastic treatment of the Wilson loop on Abelian example. First, rewrite expression for the Wilson loop:

$$
\exp \left(i \oint_{C} A_{\mu} d x_{\mu}\right)=\exp \left(-\frac{1}{2} \oint_{C} A_{\mu} d x_{\mu} \oint_{C} A_{v} d x_{v}^{\prime}\right),
$$

and, moreover:

$\exp \left(i \int_{C} A_{\mu} d x_{\mu}\right)=\exp \left(-\frac{1}{2} \iint d \sigma_{\mu v} d \sigma_{\rho \sigma}^{\prime} G_{\mu v}(x) G_{\rho \sigma}\left(x^{\prime}\right)\right)$

Now, there comes the model itself which is nothing else but assumption that the fields fluctuate independently at scale beyond the correlation length $l$. Then one has:

$$
\langle\exp \ldots\rangle=\Pi_{\Sigma \sim l^{2}} \exp (-\langle\ldots\rangle)
$$


where by the dots we denote the integrands, and the explicit expressions can be read off from (77). As a result, Eq (78)) implies:

$$
\langle W\rangle \sim \exp (-\sigma T R), \sigma \sim 1 / l^{2}
$$

where $T, R$, as usual, characterize the Wilson contour $C$. Note that in the $Z_{2}$ case the correlation length is simply the lattice spacing, see Eq. (46).

In the YM case one usually applies the same (79) and assumes soft stochastic fields:

$$
l^{2} \sim \Lambda_{Q C D}^{-2} .
$$

Then the tension is of order $\Lambda_{Q C D}^{2}$, as it should be. However, now that we know that the stochastic component of the vacuum fields is provided by infinitely thin vortices, the assumption (80) is far from being obvious.

Now, we come to a subtle point, which is actually also the central point of this subsection. The only gauge invariant correlator bilinear in non-Abelian fields is

$$
\left\langle G_{\mu \nu}^{a} \Phi_{a b}\left(x, x^{\prime}\right) G_{\mu \nu}^{b}\left(x^{\prime}\right)\right\rangle \equiv K\left(x . x^{\prime}\right)
$$

where $\Phi_{a b}\left(x, x^{\prime}\right)$ is the parallel transport from point $x$ to point $x^{\prime}$. Thus, from gauge invariance alone we can conclude that the correlator (81) enters the expression for the Wilson loop in the stochastic approximation.

Since we are working with hard gauge fields, $A \sim 1 / a$ and assume no smoothing or cooling, the self energy of the string $\Phi\left(x, x^{\prime}\right)$ is UV divergent, the same as for, say, Wilson loop. Therefore,

$$
K\left(x, x^{\prime}\right) \sim \exp \left(-\operatorname{const} \frac{\left|x-x^{\prime}\right|}{a}\right)\left\langle G^{2}\right\rangle_{\text {stochastic }},
$$

where we keep only the stochastic component of the plaquette action. Note that in the continuum limit of $a \rightarrow 0$ the exponential weight function is non-vanishing only for coinciding points, $x=x^{\prime}$.

At first sight, this observation eliminates the model itself. Note, however, that the stochastic component is represented now by the dual string and the corresponding $\left\langle G^{2}\right\rangle_{\text {stochastic }}$ is singular in the limit $a \rightarrow 0$.

It is amusing to observe that the product (82) is finite in the limit $a \rightarrow 0$ and the confining string tension appears to be in physical units. Using (68) for the $\left\langle G^{2}\right\rangle_{\text {stochastic }}$, we get numerically,

$$
\sigma_{\text {stochastic }} \approx 0.5 \sigma_{\text {total }}
$$

where by total tension we understand the string tension measured in the original $S U(2)$ theory.

Keeping in mind uncertainties of the stochastic model itself and the fact that there is no parameter to fit, Eq (83) is a remarkable success as far as numbers are concerned. Moreover, it is amusing that just singular fields allow to apply the stochastic model in the Yang-Mills case in the most transparent way.

\section{EXTRA DIMENSIONS}

It is crucial to compare the lattice findings with theoretical predictions. Theory involves strings in extra dimensions. In no way of course we could review this subject here, the references can be found in $[1,23]$. We will make only trivial remarks, simplifying as much as possible the original ideas, just to enable us to make contact with the lattice measurements.

\section{A. Running string tension}

String theory in $4 \mathrm{~d}$ is inconsistent because of the conformal anomaly. To amend the situation it was proposed to introduce 5th dimension as conjugated to energy scale [6].

The simplest way to visualize it is to think in terms of a running string tension. Namely imagine that the string tension is a function of the area itself. Then two limiting cases are easy to guess:

$$
\sigma(A) \rightarrow \infty \text { as } A \rightarrow 0(A \equiv \text { Area }) .
$$

In other words, no strings at short distances. From dimensional considerations,

$$
\sigma(A) \sim A^{-1}
$$

Another limit is the confining string:

$$
\sigma(A) \sim \Lambda_{Q C D}^{2} \text { if } A \sim \Lambda_{Q C D}^{-2}
$$

Moreover, the string tension is to reach a limiting value at scale of order $\Lambda_{Q C D}$ and not go down at larger distances

$$
\sigma(A) \sim \Lambda_{Q C D}^{2} \text { if } A \geq \Lambda_{Q C D}^{-2} .
$$

These pure qualitative considerations become a well defined framework if one assumes that the Wilson line is given by a sum over all surfaces bound by the Wilson contour $C$ allowing these surfaces to extend to the fifth dimension $z$ and evaluating the area of these surfaces with a nontrivial metrics [6]. Generically, the metrics in $5 \mathrm{~d}$ is of the form:

$$
d s^{2}=\frac{d z}{z^{2}}+a^{2}(z)\left(-d x_{0}^{2}+(d \mathbf{x})^{2}\right),
$$

where our $4 \mathrm{~d}$ space corresponds to $z=0$.

The short distance behavior (85) implies then that

$$
\lim _{z \rightarrow 0} a^{2}(z) \sim \frac{1}{z^{2}}
$$

The singularity at $z=0$ results in the singularity in self energy of heavy quarks, $M(a) \sim 1 / a$ where $a$ is the UV cut off, lattice spacing.

Existence of the limiting tension (87) implies, on then other hand, horizon $z_{\max }$ such that

$$
a^{2}\left(z_{\max }\right) \sim \Lambda_{Q C D}^{2}
$$

To further fix the form of $a(z)$ more data is needed. 
It is worth emphasizing that we are describing physics in $4 d$ in terms of $5 d$ space. Thus, nothing precludes us from checking with $4 \mathrm{~d}$ lattices predictions from theory exploiting the 5 th dimension.

So far we have discussed electric strings which can be open on the Wilson line. At short distances, however, the properties of the magnetic strings are to be similar because at short distances potential for both color and magnetic charges is Coulombic.

It is worth emphasizing that on the lattice one can study properties of strings of various lengths. Measuring nonAbelian action of finite clusters as function of their area is most straightforward. In particular, it has been demonstrated [32] that for finite clusters on average the action is considerably larger than for the infinite cluster:

$$
\begin{aligned}
(\text { Action })_{\text {finite }} \text { clusters } & \approx 0.9 \frac{\text { Area }}{a^{2}}, \\
(\text { Action })_{\text {infinite cluster }} & \approx 0.5 \frac{\text { Area }}{a^{2}},
\end{aligned}
$$

for details see the original papers [32].

To evaluate the string tension is much more difficult since it involves measuring the entropy, see Eq. (49), and it is not clear how to measure entropy of a surface populated with particles. However, it seems obvious that the entropy for smaller surfaces can only be smaller and then the observation (91) implies larger tension for smaller surfaces.

Another challenge to theory is to evaluate the spectrum of finite clusters as function of their area. The data [32] indicate a simple power law:

$$
N(A) \sim A^{-\tau}, \tau \approx 3
$$

Theoretically the index $\tau$ has not been calculated, to our knowledge.

\section{B. Further extra dimensions}

While the metrics with properties (90) is still a conjecture, the reduction of $\mathrm{N}=4$ SUSY YM to strings is well established, for review see [23]. The strings live in that case in $10 \mathrm{~d}$ space, (see Eq. (4) and put $z_{H}=0$ to address zero temperature). Further extra dimensions are compactified. The structure of extra dimensions is determined to a great extent by the symmetry of the problem.

Moreover, the Wilson line is calculable as a sum over areas of surfaces spanned on the contour $C$ with Nambu-Goto action (for details see [33]). Since the theory is conformal, the Coulomb-like potential between heavy quarks is valid at all distances.

Note that the Maldacena construction [23] is valid for a conformal theory, with no running of the coupling. The whole idea of the preceding subsection was, on the other hand, to introduce the fifth dimension as a price for the conformal anomaly in pure YM theory. Thus, there seem to be two distinct reasons to introduce extra dimensions, conformal anomaly and summation of large number of graphs. As an analogy, let me recall that divergences of perturbative expansions in field theory are also due to two different mechanisms. First, there is large number of graphs. The corresponding effect is calculable through the Lipatov's technique and accounts for no running. The other source of the factorial divergence of the coefficients is specific graphs, renormalons and the whole effect here is due to the running of the coupling. There is no technique which would allow to account for both large number of graphs and running of the coupling ${ }^{11}$.

In case of strings, it is also difficult to account both for large $N_{c}$ and running of the coupling. The most advanced work in this direction is that of Klebanov and Strassler [35] who were able to start with the Maldacena's example of N=4 SUSY YM theory and arrive at a N=1 SUSY gauge theory. The corresponding metrics can be found in the original paper.

For our purposes, it is sufficient to use the background constructed in Ref. [36] and which is in the same universality class as pure YM theory in infrared. This is a $6 \mathrm{~d}$ space $\left(x_{\mu}, z\right)$. Moreover, one of the flat and the warped coordinates are mixed up into a compactified coordinate. The resulting metrics looks as:

$$
\begin{array}{r}
d s^{2}=\frac{8 \pi}{3} \eta \lambda^{3} \sum_{i=1}^{i=4}\left(d x^{i}\right)^{2}+\frac{2 \pi}{3} \eta \lambda d \Omega_{4}^{2} \\
+\frac{8}{27} \eta \lambda \pi\left(\lambda^{2}-\lambda^{-4}\right) d \psi^{2}+\frac{8 \pi}{3} \eta \lambda\left(\lambda^{2}-\lambda^{-4}\right)^{-1} d \lambda^{2},
\end{array}
$$

where $\eta$ is a number depending on the number of colors, $x^{i}, i=$ $1,2,3,4$ is a Euclidean space and

$$
1 \leq \lambda \leq \infty, \quad 0 \leq \psi \leq 2 \pi
$$

Moreover, $\lambda \rightarrow 1$ corresponds to the infrared, or horizon while $\lambda \rightarrow \infty$ corresponds to the ultraviolet which we would like to see as our $4 \mathrm{~d}$ space.

However, the $6 \mathrm{~d}$ space (93) in the ultraviolet is becoming flat $5 \mathrm{~d}$ space. Thus, the metrics (93) does not interpolate between our space in the UV and some non-trivial geometry in the IR. One can rely still on the metrics (93) in the IR to clarify issues of the Yang-Mills dynamics at large distances.

In particular, there exists a D2 brane, with one coordinate compactified which can be open on the 't Hooft line in the UV. Since the radius of the compactified direction in (93) shrinks to zero in the infrared, the tension associated with this brane vanishes on the horizon: [37]

$$
\sigma_{\text {magnetic }}^{\text {class }}=0 \text {. }
$$

In this way one explains constructively, why the heavy monopoles are not confined (the strings which can be open on the Wilson line do not involve the compactified dimension and the corresponding tension tends to a constant in the infrared limit).

Note also that the reason for the vanishing tension (95) is of general geometric nature and is due to vanishing radius of

\footnotetext{
${ }^{11}$ For a discussion see, in particular, [34].
} 
an extra compactified dimensions. From the $4 \mathrm{~d}$ perspective, existence of compatified dimensions implies particle living on the strings (analog of Kaluza-Klein states).

Result (95) is crucial for us since it refers directly to the strings which can be detected as vacuum defects. Let us emphasize that Eq. (95) holds classically. In the classical approximation the magnetic string rests on the horizon where it becomes tensionless. Thus, nontrivial dynamic of the magnetic string arises only on the level of quantum corrections which have not been elaborated yet.

A general hint which we can extract from the idea on existence of further dimensions is that magnetic strings are populated with particles. Indeed, we have learned that magnetic strings are actually branes with some dimensions compactified. The corresponding excitations would be manifested as particles living on the strings. No detailed theory of the phenomenon is available however since the theoretical analysis so far does not go beyond the classical approximation (95).

\section{Particles living on strings}

Prediction of particles living on the strings looks very exotic and difficult to believe. Paradoxically enough, the lattice phenomenology is again ahead of theory, and such particles were observed earlier than the prediction was made. Moreover, observation of the particles even preceded historically observation of the strings themselves. However, the particles were assumed first to live in $4 \mathrm{~d}$. In the lattice terminology, we are talking about magnetic monopoles of the Maximal Abelian Projection (MAP), for review see [38]. However, in these notes we are trying to use the continuum-theory terminology and cannot give even a brief overview of the historical development. Instead, we will try to jump over to interpretation of the lattice observations.

Our problem now is to formulate notion of a particle living on a surface in terms of non-Abelian fields. The key ingredient is the requirement that the definition of particles is explicitly gauge invariant.

Let us start with similar problem for the surfaces, or strings. We have already mentioned that the non-Abelian field is aligned with the surface. This is an apparently gauge invariant statement. Consider non-Abelian fields on the surface, $G_{\mu \nu}^{a}(x)$ where point $x$ belongs to the surface and where $\mu, v$ are Lorentz indices and $a=1,2,3$ in our case. In general $G_{\mu \nu}^{a}$ represent three antisymmetric tensors, or six vectors. To enumerate independent vectors one uses chiral vector combinations, $\left(\mathbf{E}^{a} \pm \mathbf{H}^{a}\right)$ where $\mathbf{E}^{a}, \mathbf{H}^{a}$ are color electric and magnetic fields, respectively. Moreover, one can introduce determinants constructed on each triplet of the vectors, $\Delta_{ \pm}$.

For the fields to be aligned with the surface all the components $G_{\mu \nu}^{a}$ are to be reducible to two independent vectors. Gauge and rotational invariant conditions which define two vectors can be chosen, for example as

$$
\begin{gathered}
(\mathbf{E}+\mathbf{H})^{a}=0, \\
\Delta_{-} \equiv \varepsilon_{a b c} \varepsilon_{i k l}(E-H)_{i}^{a}(E-H)_{k}^{b}(E-H)_{l}^{c}=0 .
\end{gathered}
$$

Constraints on the non-Abelian fields can also be formulated in terms of the matrix $\bar{G}\left\{\begin{array}{l}a \\ \mu\end{array}\right\}\left\{\begin{array}{l}b \\ v\end{array}\right\}$,

$$
\bar{G}\left\{\begin{array}{l}
a \\
\mu
\end{array}\right\}\left\{\begin{array}{l}
b \\
v
\end{array}\right\} \equiv \varepsilon^{a b c} G_{\mu \nu}^{c},
$$

which was introduced first in attempts to construct a dual formulation of the Yang-Mills theories as a field theory again, see [39] and references therein. Introduce, furthermore, determinant of the matrix (97) in the indices $\left\{\begin{array}{l}a \\ \mu\end{array}\right\}$ and $\left\{\begin{array}{l}b \\ v\end{array}\right\}$. Then the condition

$$
\Delta\{\bar{G}\}=0,
$$

generically defines a surface in four dimensional space.

Now, definition of a particle living on the surface introduces further degeneracy of the non-Abelian fields. Namely, if the surface is determined as in (96) then particle trajectories correspond to zero of the determinant of next order:

$$
(\mathbf{E}+\mathbf{H})^{a}=0, \Delta_{-} \sim 0^{2} .
$$

The geometrical meaning of this construction is alignment of the particle trajectory and its non-Abelian field. The trajectory belongs to $2 \mathrm{~d}$ surfaces discussed above. This is 'a particle living on the string'.

\section{Abelian 'monopoles'}

Note that the field associated with the particles just introduced is in fact Abelian. Indeed, locally there is only one independent color vector associated with the particle. Imagine now that we confront the problem of searching for such particle trajectories on the lattice. One could come to the idea of projecting the original non-Abelian fields to the closest Abelian configuration. The hope is that such an abelianization of the fields does not eliminate our particles since they are Abelian in nature. The advantage of the projection is that after the projection the search for particles is easy. After the projection, non-Abelian theory is reduced to compact $\mathrm{U}(1)$ and the particles are then the monopoles discussed in some detail in section II F ${ }^{12}$.

Technically, the Abelian projection is defined as follows. First, one uses gauge-fixing freedom to minimize the functional

$$
R=\Sigma_{\text {lattice }[}\left[\left(A_{\mu}^{1}\right)^{2}+\left(A_{\mu}^{2}\right)^{2}\right]
$$

12 Historically, the search for lattice monopoles was motivated by the idea of the Abelian dominance, for review see [38]. In the Abelian projection monopoles are spherically symmetric while the non-Abelian 'particles' we are discussing now possess line-like non-Abelian field. For the first time picture with 'line-like' monopoles was introduced in Ref. [40]. These monopoles were considered however essentially artifact of the Abelian projection. Gauge-invariant nature of the 'line-like' monopoles was discussed, in particular, in Refs. [27, 41] and is not universally accepted till now. A similar picture in 3d Yang-Mills theory was verified in Ref. [42] . 
where $A_{\mu}^{a}$ is the gauge field and the indices 1,2 stand for color. Denote the potential in this gauge as $\bar{A}_{\mu}^{a}$. Then the projection is defined as neglecting the charged field altogether:

$$
\bar{A}_{\mu}^{1,2} \rightarrow 0 ;\left\{A_{\mu}^{a}\right\} \rightarrow \bar{A}_{\mu}^{3} .
$$

The monopoles are now defined as:

$$
\partial_{\mu} \bar{F}_{\mu \nu} \equiv j_{v}^{m o n}
$$

where the field strength tensor $\bar{F}_{\mu v}$ is constructed on the projected potential $\bar{A}_{\mu}^{3}$.

The definition of the monopoles might look awkward. But their properties, observed on the lattice turn to be remarkable.

\section{E. Lattice data on the monopoles}

\section{Monopole density}

First, one can measure the total length of the monopole trajectories $L_{t o t}$, or the total monopole density introduced in (54). The measurements [43] produce the following result for the monopole density:

$$
\rho_{\text {tot }}^{\text {mon }} \approx 1.6(\mathrm{fm})^{-3}+1.5(\mathrm{fm})^{-2} \cdot a^{-1}
$$

where $a$ as usual is the lattice spacing.

The data (103) is indeed remarkable since the observed density of monopoles saturates the bound derived from asymptotic freedom, see (57). The geometrical meaning of the observation (103) is that the monopoles live on $2 \mathrm{~d}$ defects.

It is worth emphasizing that the definition of the monopoles (102) in terms of the Abelian projection is not related directly to the definition of lattice strings which is given in terms of a $Z_{2}$ projection. Generically, the monopoles (103) belong to some surfaces unrelated to the lattice strings. A dramatic result is that the monopole trajectories do belong to lattice strings $[32,40]$. The evidence is pure numerical, and the fraction of the monopoles associated with the strings is typically about 90 per cent of the their total number. Moreover, their non-Abelian field is aligned with the surface [40].

Thus, there is dramatic lattice evidence that monopoles of the Abelian projection are particles living on the lattice strings.

Non-Abelian action of the monopoles

The non-Abelian action of the monopoles $S_{m o n}$ is ultraviolet divergent, at presently available lattices: [44]

$$
S_{m o n} \approx \ln 7 \frac{L_{m o n}}{a}
$$

where we quote the result in the form convenient for comparison with theory, see Eq. (16). Thus, the monopole action is tuned to the entropy. Let us emphasize again that in the nonAbelian case there is no parameter to tune since the only parameter, coupling is running. Thus, the phenomenon observed is the self tuning of a divergent action to the same divergent entropy so that the free energy is apparently finite in the limit $a \rightarrow 0$.
Note that the monopole trajectories belong to the lattice strings. Moreover, plaquettes belonging to the monopole trajectories accumulate action which is about 40 per cent higher than the action averaged over the strings. Thus the action of the magnetic strings is not simply that of Nambu-Goto, for further details see [32].

Finite monopole clusters

For finite monopole clusters one can measure the distribution in their length $N L$. The data are well fitted by: [43, 45]

$$
N(L) \sim L^{-\alpha}, \alpha \approx 3 .
$$

Note striking agreement with theoretical expectations, see Eq. (20). The effect of mass suppression at larhe $L$, predicted by the same Eq. (20) has not been seen yet ${ }^{13}$.

As for the radii of the finite cluster they satisfy

$$
r \sim \sqrt{L \cdot a}
$$

same as for random walks.

\section{Infinite cluster}

In each field configuration, there exists infinite cluster of monopole trajectories. Phenomenologically there are indications that existence of the infinite cluster is crucial for the confinement, for review see [38].

The percolating cluster exhibits remarkable scaling properties:

$$
L_{\text {perc }}^{\text {mon }} \approx 30(\mathrm{fm})^{-3} V_{\text {tot }},
$$

Historically the observation of the scaling of the infinite cluster was the first strong indication that the Abelian projection reveals existence of $\mathrm{SU}(2)$ invariant objects. Note also that relation $L_{\text {perc }} \sim \Lambda_{Q C D}^{3} V_{\text {tot }}$ is a necessary condition for the infinite cluster to be relevant to the confinement.

In section II E we also mentioned that thermodynamically one can evaluate fluctuations of the total length of the percolating cluster in the finite volume. The prediction was checked in Ref. [11] and found to agree with the data.

The result (107) can be rewritten as probability of a given link to belong to the percolating cluster:

$$
\theta_{\text {link }}^{\text {mon }} \sim\left(\Lambda_{Q C D} \cdot a\right)^{\alpha}, \alpha \approx 3
$$

Thus, we could conclude that Eq. (108) looks like a typical relation in supercritical phase, compare with Eq. (22). Eq. (108) is commonly considered as an evidence that the standard percolation picture applies to the lattice monopoles.

There is a subtle point however. In percolation theory there are various relations and inequalities between critical exponents, see, e.g., [46]. The index $\alpha$ in Eq. (108) is one of such exponents. One can demonstrate that the value of $\alpha=3$ is in contradiction with assumption that mass scales for monopoles is set by $\Lambda_{Q C D}$. Detailed derivation is beyond the scope of

\footnotetext{
13 Actually there exist long-range correlations between directions of the links along the monopole trajectory. Qualitatively, such long range correlation corresponds to a low mass scale. For details see the original papers [43].
} 
the present notes. The basic idea is easy to understand, however. The length of the percolating cluster fluctuates because finite clusters can be absorbed into the percolating cluster. The length of finite clusters, in turn, is controlled by monopole mass,

$$
L_{\text {finite }} \sim\left(m^{2} \cdot a\right)^{-1} \sim\left(\Lambda_{Q C D}^{2} \cdot a\right)^{-1}
$$

and we see that in the limit $a \rightarrow 0$ the fluctuation (109) would exceed the length of the percolating cluster itself which is not possible. This is the meaning of contradiction of the observation (108) with the standard percolation theory.

The resolution of this paradox is that monopoles actually percolate not through the whole $4 \mathrm{~d}$ space but live on two dimensional surfaces. Thus, we rewrite the exponent (108) as

$$
\alpha=2+\tilde{\alpha}^{\prime}
$$

where the term ' 2 ' reflects the constraint that particles live on a two-dimensional space. In terms of $\tilde{\alpha} \approx 1$ there is no apparent contradiction with (109). No detailed modification of the standard theory has been worked out, however, in terms of the modified indices, like $\tilde{\alpha}$.

\section{F. Conclusion on extra dimensions}

Extra dimensions can be considered as tools to describe phenomena in four dimensions. In particular, a warped fifth dimension corresponds, roughly speaking, to a running string tension. There is some support to this idea on the lattice but no dedicated study has been performed yet.

Introduction of further compact dimensions results in the conclusion that classically magnetic strings, or better to say D2 branes, are tensionless in the infrared. Hence, no area law for the 't Hooft loop. Moreover, generically, magnetic strings are to be populated with particles, which are Kaluza-Klein kind of states corresponding to a compactified dimension of the D2 brane. And, indeed, there is ample lattice evidence that the vortices are populated with particles which are nothing else but magnetic monopoles in the lattice terminology.

If we identify lattice monopoles with quanta of magnetically charged field, we can apply general relations of the polymer representation of field theory, see section II E. In particular, non-vanishing expectation value $\left\langle\phi_{M}\right\rangle$ is in one-to-one correspondence with existence of the percolating cluster. Applying Eq. (55) separately to percolating cluster and using (108) we get an estimate: [47]

$$
\left\langle\phi_{M}\right\rangle \sim\left(\Lambda_{Q C D}^{3} \cdot a\right)^{1 / 4}
$$

The vacuum expectation tends to zero in the limit $a \rightarrow 0$ while at any finite value of the lattice spacing it can be considered as an order parameter. Within string theory, observation (111) could give clue to describing tachyonic mode of a string with vanishing classical tension.

\section{TOPOLOGICAL DEFECTS IN MEASUREMENTS WITH HIGH RESOLUTION}

\section{A. Introduction}

In this section we will discuss topological defects in vacuum. By topological defects we understand regions with large absolute value of the density of the topological charge $Q_{\text {top }}(x)$,

$$
Q_{t o p}(x)=\left(16 \pi^{2}\right)^{-1} G^{a} \tilde{G}^{a} .
$$

Usually one thinks about such regions in terms of instantons. For instantons,

$$
\left(\int d^{4} x Q_{\text {top }}(x)\right)_{\text {instanton }}=1 .
$$

Moreover - and this is a crucial point for our discussion here one visualizes instantons as bulky fields of characteristic size of order $\Lambda_{Q C D}^{-1}$, see, e.g., $[48,49]$. The instanton picture has been challenged since long because of inconsistency in the large $N_{c}$ limit $[50,51]$. An alternative description could be provided by domain walls [50]. The theory of domain walls is not developed in detail, however. Note that domain walls are, by definition, $3 \mathrm{~d}$ defects in vacuum. Thus, one could argue that in the domain-walls picture topological defects would occupy a vanishing fraction of the whole $4 \mathrm{~d}$ space.

As far as we know, this point, however, has never been emphasized ${ }^{14}$. Thus, the possibility that topological defects could occupy a vanishing submanifold of $4 \mathrm{~d}$ space was suggested first in Ref [41] in the context of the 3d defects [31] discussed in section V E. Independently, there began to appear data on unusual behavior of fermionic zero modes as function of the lattice spacing [52]. The data do indicate that topological defects shrink to a vanishing subspace of the whole space. However, it is too early to conclude what is the dimension (in physical units) of this submanifold.

\section{B. Low-lying fermionic modes}

To uncover topology of the gluonic fields one concentrates on low-lying modes of the Dirac operator. The modes are defined as solutions of the eigenvalue problem

$$
D_{\mu} \gamma_{\mu} \psi_{\lambda}=\lambda \psi_{\lambda}
$$

where the covariant derivative $D_{\mu}$ is constructed on the vacuum fields $\left\{A_{\mu}^{a}(x)\right\}$.

\footnotetext{
${ }^{14}$ Locally three-dimensional structures related to the chiral symmetry breaking were introduced in [51]. However, the structures discussed in these papers do not depend on the $\Lambda_{O C D}$ and are generically the same in case of, say, photodynamics. The structures occupy (almost) the whole $4 \mathrm{~d}$ volume. In this review, we understand dimensions of the defects exclusively in the physical units and lower-dimensional defects, in our terminology, always occupy a vanishing part of the whole volume in the continuum limit.
} 
For exact zero modes, the difference between modes with positive and negative chirality equals to the total topological charge of the lattice volume:

$$
n_{+}-n_{-}=Q_{t o p} .
$$

Equation (113) can be turned into as a prediction for the average value of the topological charge squared,

$$
\left\langle Q_{\text {top }}^{2}\right\rangle \sim \Lambda_{Q C D}^{-4} V_{t o t} .
$$

The meaning of (114) is that topological charge fluctuates independently on the $4 \mathrm{~d}$ volumes measured in physical units.

One also considers near-zero modes which occupy, roughly speaking an interval

$$
0<\lambda<\frac{\pi}{L_{\text {latt }}},
$$

where $L_{\text {latt }}$ is the linear size of the lattice. Near-zero modes determine the value of the quark condensate via the BanksCasher relation:

$$
\langle\bar{q} q\rangle=-\pi \rho(\lambda \rightarrow 0),
$$

where $\lambda \rightarrow 0$ with the total volume tending to infinity.

\section{Lattice data}

While the close relation of the low-lying fermionic modes to the topology of the gluon fields is well known since long, it is only recently that these modes have been measured on the original field configurations, without cooling. The recent progress in the studying the topological fermionic modes on the lattice is due to the advent of the overlap operator [53].

Measurements [52] confirm validity of the general relations (114) and (116). However, they also bring an unexpected result that the volume occupied by low-lying modes apparently tends to zero in the continuum limit of vanishing lattice spacing, $a \rightarrow 0$. Namely,

$$
\lim _{a \rightarrow 0} V_{\text {mode }} \sim\left(a \cdot \Lambda_{Q C D}\right)^{r} \rightarrow 0,
$$

where $r$ is a positive number of order unit and the volume occupied by a mode, $V_{\text {mode }}$ is defined in terms of the Inverse Participation Ratio (IPR) ${ }^{15}$.

A crucial question is then, whether the underlying vacuum structure is the same for the confining fields and fields with non-trivial topology. An attempt to answer this question was undertaken in Ref. [55] through a direct study of correlation between intensities of fermionic modes and of vortices.

In more detail, center vortex is a set of plaquettes $\left\{D_{i}\right\}$ on the dual lattice. Let us denote a set of plaquettes dual to $\left\{D_{i}\right\}$ by $\left\{P_{i}\right\}$. Then the correlator in point is defined as:

$$
C_{\lambda}(P)=\frac{\sum_{P_{i}} \sum_{x \in P_{i}}\left(\rho_{\lambda}(x)-\left\langle\rho_{\lambda}(x)\right\rangle\right)}{\sum_{P_{i}} \sum_{x \in P_{i}}\left\langle\rho_{\lambda}(x)\right\rangle} .
$$

\footnotetext{
15 Independent evidence in favor of shrinking of the regions occupied by topologically non-trivial gluon fields was obtained in [54].
}

Since $\sum_{x} \rho_{\lambda}(x)=1$ and $\left\langle V_{\text {tot }} \rho_{\lambda}(x)\right\rangle=1$, Eq (81) can be rewritten as

$$
C_{\lambda}(P)=\frac{\sum_{P_{i}} \sum_{x \in P_{i}}\left(V_{t o t} \rho_{\lambda}(x)-1\right)}{\sum_{P_{i}} \sum_{x \in P_{i}} 1} .
$$

Results of measurements can be found in the original paper [55]. Here we just briefly summarize the finding. There is a strong positive correlation between intensities of topological modes and density of vortices nearby. Moreover, the value of the correlator depends on the eigenvalue and the correlation is strong only for the topological fermionic modes.

Finally and most remarkably, the correlation grows with diminishing lattice spacing. A simple analysis demonstrates that if the $2 \mathrm{~d}$ defects are either entirely responsible for chiral symmetry breaking or constitute a boundary of $3 \mathrm{~d}$ defects carrying large topological charge, the correlator (118) grows as an inverse power of the lattice spacing. The data does show that the correlator grows for smaller $a$ but does not allow to fix uniquely the dimensionality of the chiral defects.

\section{Field-theoretic arguments}

The result (117) is in striking contradiction with the instanton model and at first sight seems very difficult to appreciate. A more careful analysis demonstrates, however, that the shrinking of topological fermionic modes could have predicted from field theory 16 .

The point is that the lattice spacing should be treated now not so much as ultraviolet cut off needed to make sense of field theory but rather as resolution of measurements. An observation is then that there exist measurements whose results depend crucially on the resolution. In case of quantum mechanics, such an example is provided by instantaneous velocity. In case of field theory we should also distinguish between matrix elements protected and unprotected against effects of high resolution.

In particular, the quark condensate (116) is expressed in the following way:

$$
\langle\bar{q} q\rangle \sim \lim _{m \rightarrow 0} m \cdot \int d \lambda \frac{\rho(\lambda)}{\lambda^{2}+m^{2}},
$$

where $\rho(\lambda)$ is the density of states with given eigenvalue $\lambda$. With improving resolution, $a \rightarrow 0$ the number of fermionic modes grows and integration in (120) extends further and further into the ultraviolel. However, it is obvious that in the chiral limit, $m \rightarrow 0$ all these extra modes do not contribute to the matrix element.

To relate the size of instanton to a matrix element we could try a non-local generalization of the quark condensate. Because of the gauge invariance, however, we should introduce then the string, same as in (82):

$$
\langle\bar{q} q\rangle_{\text {non-local }}=\langle\bar{q}(x) K(x, 0) q(0)\rangle .
$$

\footnotetext{
16 The argumentation was worked out by A.I. Vainshtein and the author and
} outlined in some detail in the talk [56]. 
Substituting soft instanton fields into (121) we would get a non-vanishing result for finite $x$. However, as we already discussed, with improving resolution, $a \rightarrow 0$, the factor $K(x, o)$ shrinks to delta-function. Thus, the non-local matrix element (121) cannot be defined in a way independent on details of the measurement procedure.

Similar arguments can be given in terms of the gluon fields alone. Indeed, the fermionic modes just reveal the topological structure of the underlying gluon fields. Consider correlator of topological densities at two points. From general principles alone, one can derive:

$$
\begin{gathered}
\langle G \tilde{G}(x), G \tilde{G}(0)\rangle_{\text {Minkowski }}>0 \\
\langle G \tilde{G}(x), G \tilde{G}(0)\rangle_{\text {Euclidean }}<0 .
\end{gathered}
$$

On the other hand, for an instanton, or within a zero mode

$$
\langle G \tilde{G}(x), G \tilde{G}(0)\rangle_{\text {instanton }}<0 .
$$

Since the general relations (122) are based on unitarity alone contribution (123) which we are looking for, is anti-unitary.

Since the instanton contribution (123) taken alone violates unitarity, it cannot dominate at any finite $x$ and the unitarity is restored by perturbative contributions. Somewhat schematically, the correlator can be represented as

$$
\langle G \tilde{G}(x), G \tilde{G}(0)\rangle_{\text {Euclidean }} \sim-\frac{c_{1} \alpha_{s}^{2}}{x^{8}}+c_{2} \Lambda_{Q C D}^{4} \delta(x),
$$

where $c_{1,2}$ are positive constants and $1 / x^{8}$ is perturbative.

The central point is that by measuring topological modes we filter the perturbative noise away and are left with the local term in (124). In the language of dispersion relations, this is a subtraction term, which has no imaginary part [57].

It is only natural then that contributions which are described by subtraction constants in dispersion relations appear as vanishing sub-manifolds once attempt is made to measure their spatial extension, or volume. Moreover, to see that the volume is small measurements with high resolution are needed. This explains dependence on the lattice spacing exhibited by the data (117).

Although this type of argument makes observation (117) absolutely natural and predictable, it does not immediately fix the exponent $r$. Using an analogy with quantum mechanics, one can argue that $r=1$, for details see [56].

While the shrinking of topological modes (117) follows from Yang-Mills theory, explaining the observed correlation of the topological modes with the lattice strings is beyond the scope of field theory. Probably, clues are provided by theory of the defects in the dual, string formulation but there has been no discussion of the issue in the literature.

\section{CONCLUSIONS}

Confinement and chiral symmetry breaking have been discussed theoretically for many years exclusively in terms of bulky fields, with size of order $\Lambda_{Q C D}^{-1}$. Discovery of vacuum defects of lower dimension in lattice Yang-Mills theory came as a full surprise. A setback for appreciation of the meaning of this discovery is that original definitions of the defects are given in specific lattice language and appear to be not unique at that. However, the properties of the defects are SU(2) invariant and it is becoming more and more obvious that by means of projections one is observing true $\mathrm{SU}(2)$ invariant objects, magnetic strings and, possibly, domain walls. The latest evidence of this type is observation of strong correlation between magnetic strings and intensity of topological modes, defined in explicitly covariant way [55].

Independently, magnetic strings and topology-related domain walls were introduced in dual formulations of gauge theories. However, the dual representations are derived in the limit of large number of colors and it is far from being obvious that the results apply to the $\mathrm{SU}(2)$ or $\mathrm{SU}(3)$ cases. The assumption that the basic geometrical constructions survive even if $N_{c}$ is not large provides a phenomenological framework known as AdS/QCD correspondence, for review see, e.g., [2].

In this review, we tried to bridge lattice data and the continuum theory involving strings living in extra dimensions. Phenomenologically, there is some support to the idea that basic features of the lattice strings and of dual, or magnetic strings of the continuum theory are similar. In particular, description of the strings in the continuum in terms of extra compactified dimensions leads generically to prediction of Kaluza-Klein excitations, or particles living on the strings. On the lattice excitations of this type were indeed observed. The wrapped fifth dimension implies running of the string tension as function of its length. There is some evidence for such a running in the lattice data but much more could have been done both on the lattice and continuum sides to check this idea.

If further data does not disprove reality of the lattice strings, the very existence of the lattice strings provides a strong evidence in favor of the AdS/QCD correspondence. It is most remarkable that the lattice data refer to a fully quantum version of the theory. Moreover, lattice data may favor certain schemes of the AdS/QCD correspondence. In particular, inclusion into the metrics of quadratic terms seem to be required by the lattice data [58].

Lattice strings can also clarify the microscopic nature of dimension two gluon condensate, $\left\langle A_{\mu}^{2}\right\rangle_{\min }[59,60]$. The point is that, generically, the gauge potential in non-Abelian case can be expressed in terms of the field-strength tensor and its (ordinary) derivatives: ${ }^{17}$

$$
A=\frac{1}{g}(\partial \bar{G})(\bar{G})^{-1},
$$

where the matrix $\bar{G}$ is introduced in Eq. (97). However, the strings correspond to zeros of the determinant of the matrix $\bar{G}$ (see subsection VIC) and the matrix $(\bar{G})^{-1}$ is not defined. Thus, along the strings the gauge potential is not reducible to the field-strength tensor. Although the strings occupy a fraction of the total $4 \mathrm{~d}$ volume which vanishes in the continuum

\footnotetext{
${ }^{17}$ For details and references see [39].
} 
limit, the corresponding value of $\left\langle A_{\mu}^{2}\right\rangle_{\min }$ is of order $\Lambda_{Q C D}^{2}$ because of the singular nature of the fields associated with the string and can be relevant to confinement.

\section{Acknowledgments}

I am thankful to the organizers of the Conference "Infrared QCD in Rio", and especially to S.P. Sorella for the invitation and hospitality. I am thankful to participants of the Confer- ence, and especially to D. Dudal, M. Schaden,and H. Verschelde for the interest in the talks and discussions.

I am thankful to members of the ITEP lattice group, and especially to M.N. Chernodub, F.V. Gubarev, A.V. Kovalenko, S.M. Morozov, M.I. Polikarpov, and S.N. Syritsyn for sharing their data with me and numerous detailed discussions.

I would also like to acknowledge useful discussions with O. Andreev, A. Di Giacomo, J. Greensite, I.R. Klebanov, G. Marchesini, A.M. Polyakov, V.A. Rubakov, E.V. Shuryak, and A.I. Vainshtein.
[1] I. R. Klebanov, " $Q C D$ and string theory", [arXiv:hep$\mathrm{ph} / 0509087]$.

[2] J. Erdmenger, N. Evans, and J. Grosse, "Heavy-light mesons from the AdS/CFT correspondence" [arXiv:hep-th/0605241].

[3] K. Peeters, J. Sonnenschein, and M. Zamaklar, "Holographic melting and related properties of mesons in a quark gluon plasma", [arXiv:hep-th/0606195].

[4] D. Tong, " TASI lectures on solitons: Instantons, monopoles, vortices and kinks", [arXiv:hep-th/0509216].

[5] S. S. Gubser, "Drag force in AdS/CFT "[arXiv:hepth/0605182];

J.J. Friess, S. S. Gubser, and G. Michalogiorgakis, "Dissipation from a heavy quark moving through $N=4$ super-Yang-Mills plasma", [arXiv: hep-th/0605292].

[6] A.M. Polyakov, Phys. Lett. B 103, 207 (1981); A. M. Polyakov, Int. J. Mod. Phys. A 14, 658 (1999), [arXiv:hep-th/9809057].

[7] J. Greensite, Progr. Part. Nucl. Phys. 51, 1 (2003), [arXiv:heplat/0301023].

[8] M.N. Chernodub, F.V. Gubarev, M.I. Polikarpov, and V. I. Zakharov, Phys. Atom. Nucl. 64, 561 (2001), Yad. Fiz. 64, 615 (2001), [arXiv:hep-th/0007135].

[9] A. M. Polyakov, Phys. Lett. B 59, 82 (1975).

[10] A.M. Polyakov, "Gauge Fields and Strings", Harvard Academic Publishers, (1987);

J. Ambjorn, "Quantization of geometry", [arXiv:hepth/9411179].

[11] K. Ishiguro, M.N. Chernodub, K. Kobayashi, and T. Suzuki, Nucl. Phys. Proc. Suppl. 129, 659 (2004), [arXiv:heplat/0308004]

[12] M.E. Peskin, Annals Phys. 113, 122 (1978).

[13] M. Panero, JHEP 0505, 066 (2005), [arXiv:hep-lat/0503024].

[14] H. Shiba, T. Suzuki, Phys. Lett. B 343, 315 (1995), [arXiv:heplat/9406010].

[15] Th. A. DeGrand, D. Toussaint , Phys. Rev. D 22, 2478 (1980).

[16] R. Savit, Rev. Mod. Phys. 52, 453 (1980).

[17] A. Di Giacomo, H.G. Dosch, V.I. Shevchenko, and Yu.A. Simonov, Phys. Rept. 372, 319 (2002), [arXiv:hep-ph/0007223].

[18] A.V. Kovalenko, (2005) unpublished.

[19] V.S. Dotsenko, P. Windey, G. Harris, E. Marinari, E. J. Martinec, and M. Picco, Phys. Rev. Lett. 71, 811 (1993), [arXiv:hep-th/9304088].

[20] V.I. Zakharov, AIP Conf. Proc. 756, 182 (2005), [arXiv:hep$\mathrm{ph} / 0501011]$

[21] V.I. Zakharov, "Nonperturbative match of ultraviolet renormalon", [arXiv:hep-ph/0309178].

[22] M. Beneke, V.I. Zakharov, Phys. Rev. Lett. 69, 247 (1992).

[23] O. Aharony, S. S. Gubser, J. M. Maldacena, H. Ooguri, and Y.
Oz, Phys. Rept. 323, (2000), [arXiv:hep-th/9905111].

[24] G. 't Hooft, Nucl. Phys. B 138, 1 (1978).

[25] M.N. Chernodub F.V. Gubarev, M.I. Polikarpov, and V.I. Zakharov, Phys. Lett. B 514, 88 (2001), [arXiv:hep-ph/0101012].

[26] L. Del Debbio, M. Faber, J. Greensite, and S. Olejnik, Phys. Rev. D 55, (1997) 2298, [arXiv:hep-lat/9610005].

[27] F. V. Gubarev,A. V. Kovalenko, M. I. Polikarpov, S. N. Syritsyn, and V. I. Zakharov, Phys. Lett. B 574, 136 (2003), [arXiv:heplat/0212003].

[28] M. Faber, J. Greensite, S. Olejnik, and D. Yamada, JHEP 9912, 012 (1999), [arXiv:hep-lat/9910033].

[29] A. M. Polyakov, Phys. Lett. B 72, 477 (1978).

[30] P. de Forcrand, M. D’Elia, Phys. Rev. Lett. 82, 4582 (1999), [arXiv:hep-lat/9907028].

[31] A.V. Kovalenko, M.I. Polikarpov, S.N. Syritsyn, and V.I. Zakharov, Phys. Lett. B 613, 52 (2005), [arXiv:hep-lat/0408014]; M.I. Polikarpov, S.N. Syritsyn, and V.I. Zakharov, JETP Lett. 81, 143 (2005) 143, [arXiv:hep-lat/0402018].

[32] A. V. Kovalenko, M. I. Polikarpov, S. N. Syritsyn, and V. I. Zakharov, Phys. Rev. D 71, 054511 (2005), [arXiv:heplat/0402017];

A. V. Kovalenko, M. I. Polikarpov, S. N. Syritsyn, and V. I. Zakharov, Nucl. Phys. Proc. Suppl. 129, 665 (2004), [arXiv:heplat/0309032].

[33] J.M. Maldacena, "Wilson loops in large N field theories.”, Phys. Rev. Lett. 80, 4859 (1998), [arXiv:hep-th/9803002];

Soo-Jong Rey, Jung-Tay Yee, Eur. Phys. J. C 22, 379 (2001), [arXiv:hep-th/9803001].

[34] A.I. Vainshtein, V.I. Zakharov, Phys. Rev. Lett. 73, 1207 (1994), [arXiv:hep-ph/9404248].

[35] I.R. Klebanov, M. J. Strassler, JHEP 0008, 052 (2000), [arXiv:hep-th/0007191]

[36] E. Witten, Adv. Theor. Math. Phys. 2, 505 (1998), [arXiv:hepth/9803131].

[37] N. Itzhaki, J.M. Maldacena, J. Sonnenschein, and S. Yankielowicz, Phys. Rev. D 58, 046004 (1998), [arXiv:hepth/9802042];

D. Gross, H. Ooguri, Phys. Rev. D 58, 106002 (1998), [arXiv:hep-th/9805129];

A. Armoni, E. Fuchs, and J. Sonnenschein, JHEP (1999) 9906 , [arXiv:hep-th/9903090].

[38] M.N. Chernodub, F.V. Gubarev, M.I. Polikarpov, and A.I. Veselov, Prog. Theor. Phys. Suppl. 131, 309 (1998), [arXiv:hep-lat/9802036];

A. Di Giacomo, Prog. Theor. Phys. Suppl. 131, 161 (1998), [arXiv:hep-lat/9802008];

T. Suzuki, Prog. Theor. Phys. Suppl. 131, 633 (1998).

[39] O. Ganor, J. Sonnenschein, Int. J. Mod. Phys. A 11, 5701 
(1996), [arXiv:hep-th/9507036].

[40] L. Del Debbio, M. Faber, J. Greensite, and S. Olejnik, "Center dominance, center vortices, and confinement", In *Zakopane 1997, New developments in quantum field theory* $p$. 47, [arXiv:hep-lat/9708023];

J. Ambjorn, J. Giedt, J. Greensite, and S. Olejnik, JHEP 0002, 033 (2000), [arXiv:hep-lat/9907021].

[41] V.I. Zakharov, Yad. Fiz. 68, 603 (2005), [arXiv:hep$\mathrm{ph} / 0410034]$.

[42] M. N. Chernodub, R. Feldmann, E.-M. Ilgenfritz, and A. Schiller, Phys. Lett. B 605, 161 (2005), [arXiv:heplat/0406015].

[43] P.Yu. Boyko, M.I. Polikarpov, and V.I. Zakharov, Nucl. Phys. Proc. Suppl. 119, 724 (2003), [arXiv:hep-lat/0209075];

V.G. Bornyakov, P.Yu. Boyko, M.I. Polikarpov, and V.I. Zakharov, Nucl. Phys. B 672, 222 (2003) , [arXiv:heplat/0305021].

[44] V.G. Bornyakov, et al., Phys. Lett. B 537, 291 (2002), [arXiv:hep-lat/0103032]

[45] A. Hart, M. Teper, Phys. Rev. D 60, 114506 (1999), [arXiv:hep-lat/9902031].

[46] G. Grimmelt, "Percolation”, Berlin, Springer , (1999), Grundlehren der mathematischen Wissenschaften, vol. 321.

[47] M.N. Chernodub, V.I. Zakharov, Nucl. Phys. B 669, 233 (2003), [arXiv:hep-th/0211267].

[48] Th. Schafer , E.V. Shuryak, Rev. Mod. Phys. 70, 323 (1998), [arXiv:hep-ph/9610451].

[49] M. Teper, Nucl. Phys. Proc. Suppl. 83, 146 (2000), [arXiv:heplat/9909124].

[50] E. Witten, "Instantons, The Quark Model, And The 1/N Expansion”, Nucl. Phs., B 145, 110 (1978);

E. Witten, Phys. Rev. Lett. 81, 2862 (1998), [arXiv:hepth/9807109].

[51] I. Horvath, et al., Phys. Rev. D 67, 011501 (2003), [arXiv:heplat/0203027];

I. Horvath, et al., Phys. Lett. B 612, 21 (2005), [arXiv:heplat/0501025];

H.B. Thacker, PoS LAT2005, 324 (2006), [arXiv:heplat/0509057].

[52] C. Aubin, et al. "The Scaling Dimension of Low Lying Dirac Eigenmodes And Of The Topological Charge Density", [arXiv:hep-lat/0410024];
F.V. Gubarev, S.M. Morozov, M.I. Polikarpov, and V.I. Zakharov, JETP Lett. 82, 343 (2005), [arXiv: hep-lat/0505016]; " Localization of low lying Eigenmodes for chirally symmetric Dirac operator”, PoS LAT2005:143,2005, [arXiv:heplat/0510098];

Y. Koma et al., "Localization properties of the topological charge density and the low lying eigenmodes of overlap fermions", PoS LAT2005:300,2005, [arXiv:hep-lat/0509164];

V. Weinberg, E.-M. Ilgenfritz, K. Koller, Y. Koma, G. Schierholz, T. Streuer. " The QCD vacuum probed by overlap fermions", [arXiv:hep-lat/0610087];

C. Bernard et al., "More evidence of localization in lowlying Dirac spectrum” PoS LAT2005:299,2005, [arXiv:heplat/0510025].

[53] H. Neuberger, B 417, 141 (1998), [arXiv:hep-lat/9707022]; Phys. Lett. B 427, 353 (1998), [arXiv:hep-lat/9801031].

[54] P.Yu. Boyko, F.V. Gubarev, and S.M. Morozov, Phys. Rev. D 73, 014512 (2006), [arXiv:hep-lat/0511050].

[55] A.V. Kovalenko, S.M. Morozov, M.I. Polikarpov, and V.I. Zakharov, "On topological properties of vacuum defects in lattice Yang-Mills theories”, [arXiv:hep-lat/0512036].

[56] V.I. Zakharov, " Matter of resolution: From quasiclassics to fine tuning”, [arXiv:hep-ph/0602141].

[57] M. Aguado, E. Seiler, Phys. Rev. D 70, 107706 (2004), [arXiv:hep-lat/0406041].

[58] O. Andreev, " $1 / q^{* * 2}$ corrections and gauge/string duality", Phys. Rev. D73 (2006) 107901,[arXiv:hep-th/0603170]; O. Andreev, V.I. Zakharov, Phys. Rev. D 74, 025023 (2006), [arXiv:hep-ph/0604204].

[59] F.V. Gubarev, L. Stodolsky, and V.I. Zakharov, Phys.Rev.Lett. 86, 2220 (2001), [arXiv:hep-ph/0010057];

F.V. Gubarev, V.I. Zakharov, Phys. Lett. B 501, 28 (2001), [arXiv:hep-ph/0010096];

K.-I. Kondo, Phys. Lett. B 514, 335 (2001), [arXiv:hepth/0105299].

[60] H. Verschelde, K. Knecht, K. Van Acoleyen, and M. Vanderkelen, Phys. Lett. B 516, 307 (2001), [arXiv:hep-th/0105018]; D. Dudal, R.F. Sobreiro, S.P. Sorella, and H. Verschelde, Phys. Rev. D 72, 014016 (2005), [arXiv:hep-th/0502183]; S.P. Sorella, Annals Phys., 321, 1747 (2006). 